\title{
PEINTURES DE SECOND STYLE "SCHÉMATIQUE » EN GAULE ET DANS L'EMPIRE ROMAIN
}

\author{
par Alix BARBET
}

En élaborant le catalogue des peintures murales de Glanum ${ }^{1}$, nous nous sommes aperçue que des peintures à fond blanc, ornées de hampes à volutes noires, qui semblaient ne se rattacher à rien, faisaient au contraire partie de tout un ensemble de documents qui ont toujours embarrassé leurs inventeurs. En effet, ce type de décor est pauvre en couleurs et en motifs; on aurait pu croire qu'il était trop médiocre, trop peu caractéristique pour être daté. Fort heureusement, il n'en est rien et la confrontation de tous les exemplaires, avec leurs repères chronologiques, lorsqu'ils existent, nous ont amenée à penser qu'il s'agissait en fait d'un Second style de caractère "schématique ». Glanum nous a fourni deux spécimens de cette décoration, d'une part dans la maison "aux deux alcôves " (XVIII), d'autre part sur un mur situé à l'intérieur du péribole des temples géminés (XXIII et XXIV), sous le niveau de Glanum III².

\section{La Maison aux deux alcôves (XVIII).}

Seules deux pièces contiennent des peintures murales à leur partie inférieure. Elles sont en enfilade et communiquent entre elles par une petite porte (fig. 1). Les murs, en partie fondés sur les restes de constructions de Glanum I, donnent une datation précieuse : outre ces vestiges de Glanum I réutilisés, qui indiquent que la construction de la maison se place forcément après la fin du II ${ }^{\mathrm{e}}$ siècle av. J.-C., le reste de la maçonneric en moellons irréguliers, hourdés en mortier de terre, est de même nature que celle qui est employée dans la maison de Sulla (XII) : en opus incerlum. Ce petit appareil ne s'élève qu'à une hauteur moyenne de $80 \mathrm{~cm}$, au-dessus la construction en argile crue s'est délitée.

La maison aux deux alcôves (XVIII) a été recouverte par le monument à piliers de

(1) A. BARBEt, Recherches sur les peintures murales de Glanum, calalogue raisonné, thèse de doctorat de $3^{e}$ cycle non publiée.

(2) H. Rolland, Fouilles de Glanum (St-Rémy-de-Provence), ler supplément à Gallia, Paris, 1946; Fouilles de Glanum, 1946-1956, XI' supplément à Gallia, 1958; Fouilles de Glanum 19.56-19.57, dans Gallia, XVI, 1958, p. 95-114 : la maison que nous avons appelée ‘aux deux alcỏves ", à cause de son plan, est dénommée dans le rapport : - maison aux enduits peints ". 


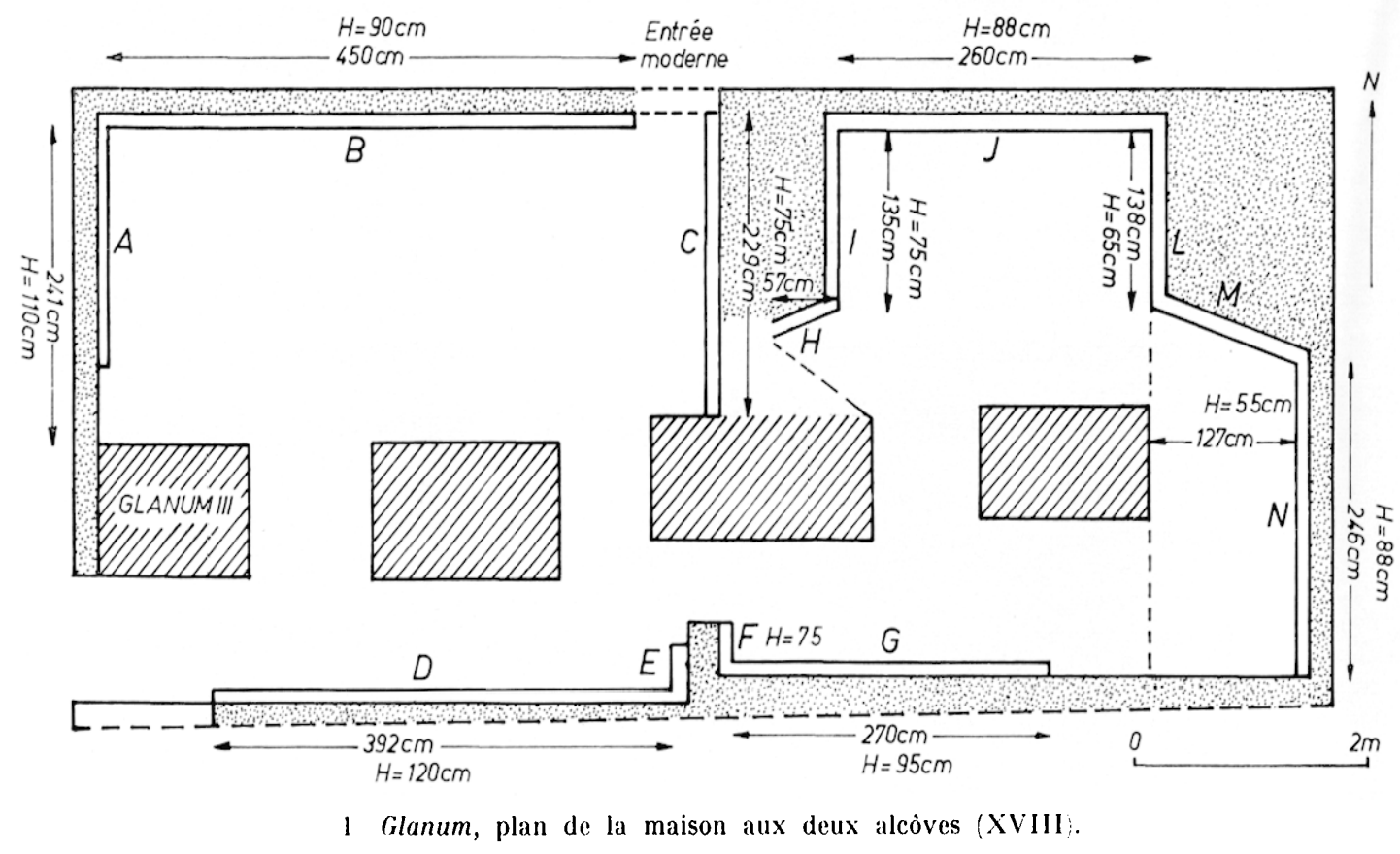

Glanum III, dont les piles de fondation d'un premier projet, abandonné en cours d'exécution, reposent sur le sol de l'habitation ${ }^{3}$. Elle appartient par conséquent à la période précédente, c'est-à-dire à Glanum II, comme le prouvent le type d'appareil employé et les remplois de grand appareil de Glanum I. Les très rares débris de céranique d'Arezzo, appartenant au début de la fabrication, prouvent que l'occupation de la maison s'est prolongée jusqu'aux environs de 30 à 20 av. J.-(. , immédiatement avant l'établissement de la terrasse inféricure de Glanum III.

Il y a un contraste frappant entre les peintures de la première salle, à hampes à volutes ténues sur fond blanc, et les peintures de la seconde salle, dont les parties supérieures sont riches en couleurs et en sujets inspirés des architectures peintes du Second style de Pompéi. seules les premières seront étudićes ici.

La décoration de la première salle est simple ; les murs $\mathrm{A}, \mathrm{B}, \mathrm{C}, \mathrm{D}$ ont la même plinthe d'un blanc uni, dù à la couleur même de l'enduit. Elle est divisée, de $70 \mathrm{~cm}$ en $70 \mathrm{~cm}$ en moyenne, par des hampes verticales noires, jaillissant du sol, surmontées d'un motif à deux volutes. Entre les deux volutes recourbées, au centre, un petit bulbe s'élive; sous les volutes, deux petites barbes latérales s'atlachent à la hampe (fig. 2 et 3). Les volutes sont de tracé inégal, celle de droite est en génćral mieux venue, plus grosse que celle de gauche. Ce détail est bien visible sur le mur $\mathrm{A}$, il prouve que le peintre était droitier et n'utilisait pas de patron lui permettant d'atteindre à une symétrie parfaite.

Au-dessus de cette plinthe, limitée par un filet rouge, à distances presque égales des hampes qui servent d'axe de symétrie, se dressent des plaques rectangulaires très étroites, entourées de filets noirs et rouges. Le fond de l'enduit est toujours blanc. Ces plaques séparent de grands compartiments rectangulaires les uns des autres. Sur le mur sud (D), en meilleur élat (hauteur de l'enduit : 1, 20 m) ill-dessus de la quatrième hampe à volutes (en commençant par la gauche), le décor change : au

(3) H. Rolland, Gallia, XVI, 1958, p. 107 et s. 


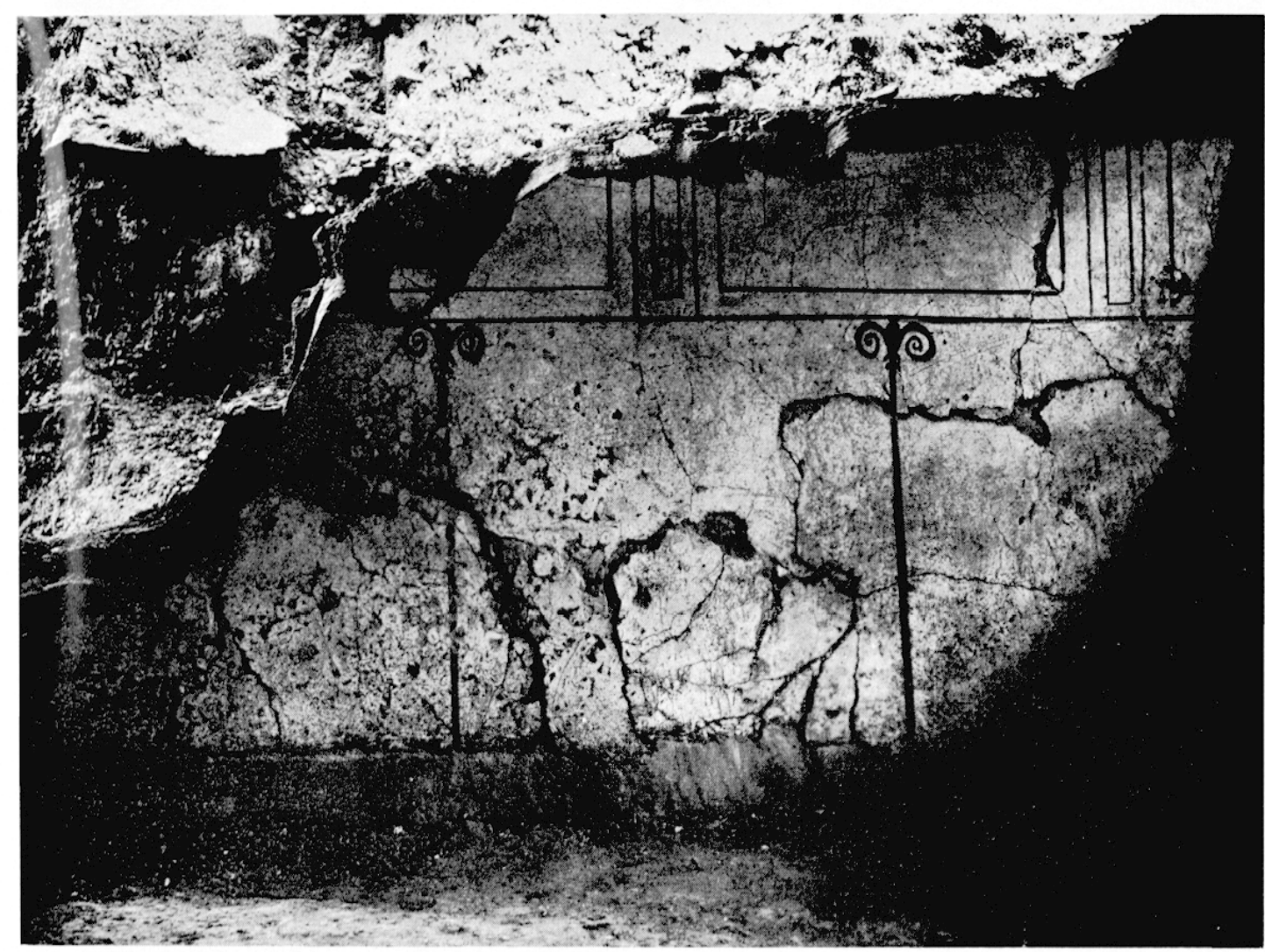

2 Glanum, maison aux deux alcôves (XVIII), mur A.

lieu d'un compartiment rectangulaire, les filets noirs et rouges dessinent un triangle reposant sur la pointe 4 . Le motif devait être un losange, dont deux còtés seulement ont subsisté, comme nous le proposons sur la restitution graphique (fig. 4).

Quelques fragments de ce décor de hampes, de volutes et de compartiments tracés par des lignes rouges et noires sont conservés au musée de St-Rémy (catalogue, nos 1 à 8). Certains, même, présentent des espacements de lignes qui ne s'accordent pas avec la décoration attestée sur les murs (catal. nos $6,9 \mathrm{a}$ ). Il faut supposer qu'ils faisaient partie d'un décor différent, audessus du précédent, peut-être d'un système de hloc's d'appareil isorlome à bossages

4) H. Rolsavi, rallia, XVI, 1958, p. 109, fig. 9 .

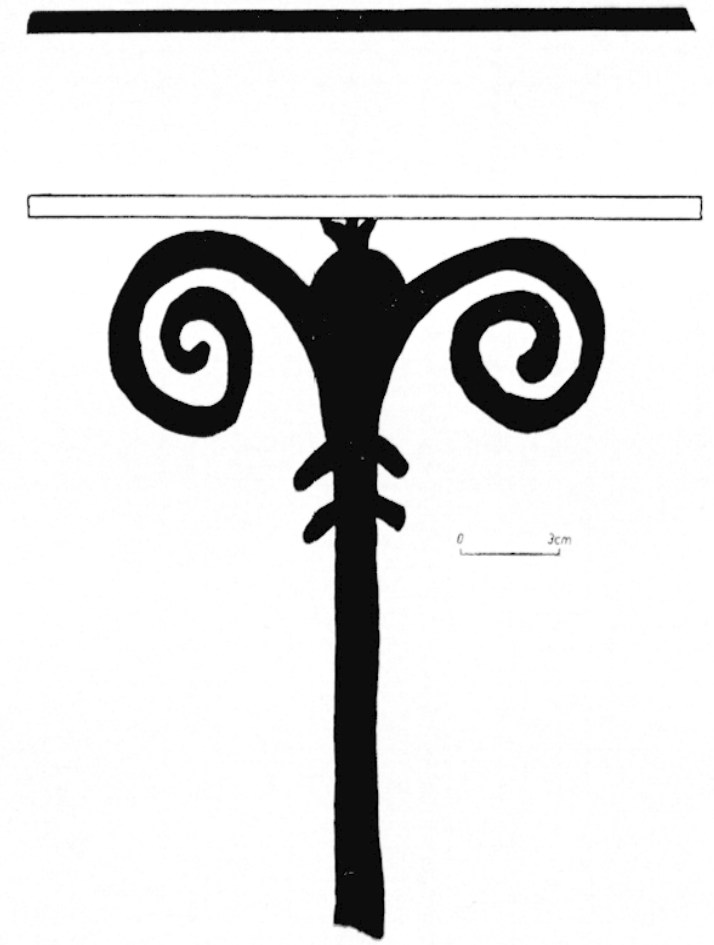

3 Glanum, maison allx deux alcòves (XVIIl), detail de la volute d'extrême droite du mur D. 


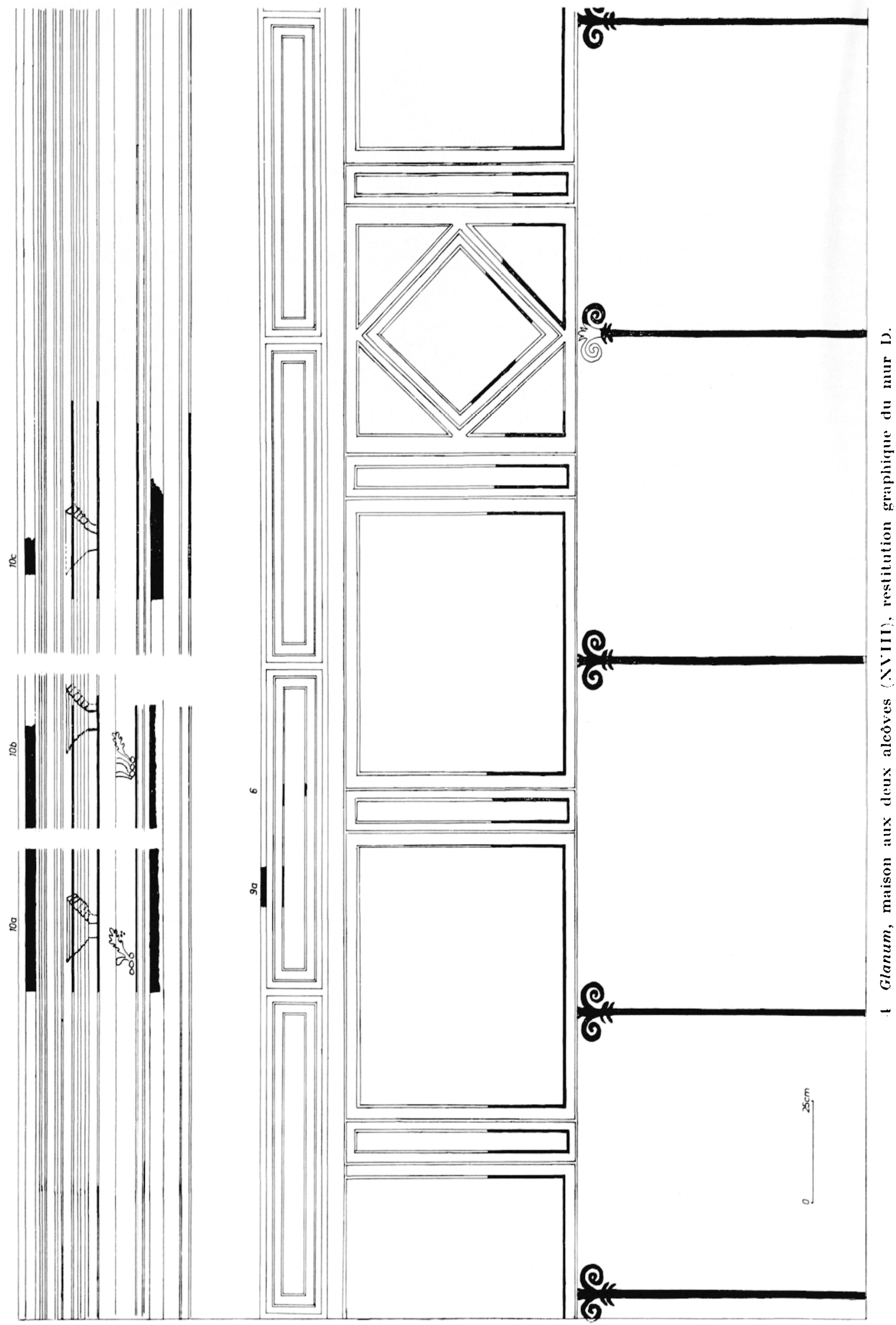




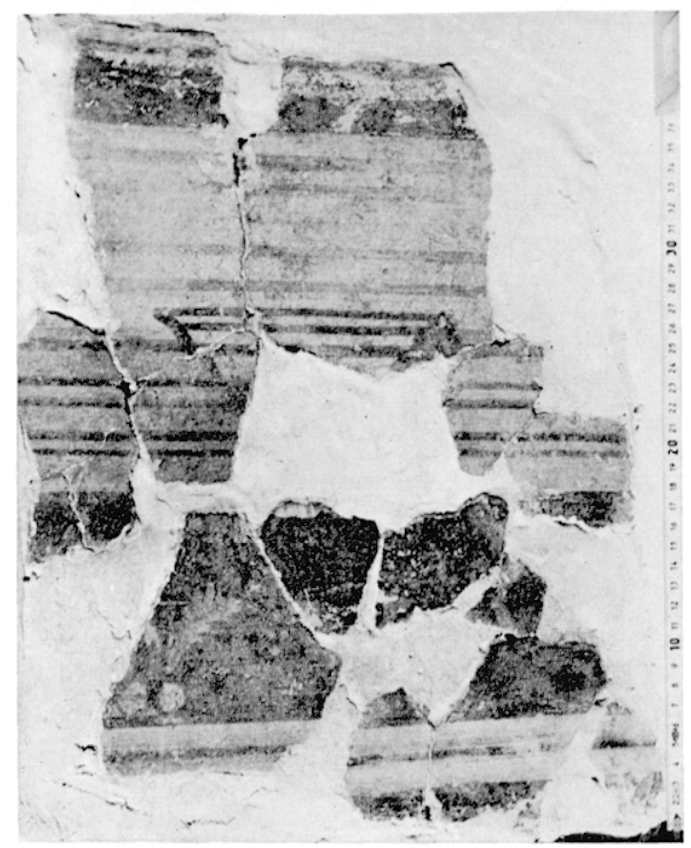

5 Glanum, maison aux deux alcòves (XVIII), fragment d'entablement (catal. no 10 a).

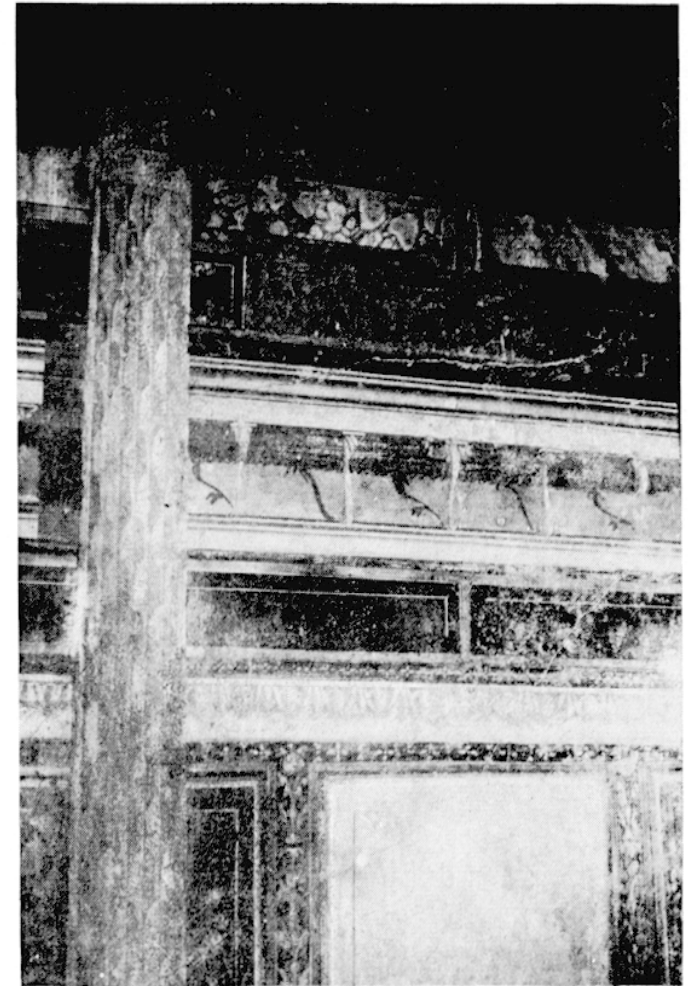

6 Pompéi, maison des Noces d'argent Région $V$, insula 2 , E), salle $\mathrm{Z}$, mur du fond.

peints, cormme nous le suggérons par comparaison avec une peinture analogue trouvée sur le Palatin (fig. 22).

Le sommet des murs de la maison, reconstitué en petits panneaux au musée de St-Rémy (catal. nos $10 \mathrm{a}, 10 \mathrm{~b}, 10 \mathrm{c}$ ), montre que l'enduit était blanc jusqu'en haut et qu'une sorte d'entablement architectural avec corniche à moulures, frise rouge bordeaux et consoles, était tracé en lignes brunes légères. La longueur conservée de cet entablement dépasse $3,50 \mathrm{~m}$ (fig. 5).

Ces consoles ont une forme caractéristique que nous retrouvons très souvent à Pompéi et sur des peintures qui figuraient au sommet d'un mur de la maison de Sulla (XII) à Glanum, où elles supportent des modillons en forme de S. Elles sont "suspendues" et ne supportent pas de façon convaincante la corniche moulurée. Aucun caisson vu en perspective n'est représenté, comme c'est le cas dans la peinture romaine où les consoles (parfois sans modillons) soutiennent une corniche vue en profondeur, dont les caissons sont bien apparents. Pour ne prendre qu'un exemple, la maison des Griffons sur le Palatin en montre à plusieurs endroits.

Autre inconséquence de la part du peintre de Glanum: à la base de la frise rouge bordeaux, des petites boules et un motif en forme de feuille blanche qui représentent le départ de modillons et leur ombre portée ne sont pas situés juste sous la console, comme la logique le voudrait. Une comparaison avec une peinture de la maison des Noces d'argent 
(Région $\mathrm{V}$, Insula $2, \mathrm{E}$ ) à Pompéi fera sentir toute la différence qui sépare une peinture du Second style pompéien coloré et ce style schématique de Glanum (fig. 6). L'ombre portée, au lieu d'être plus sombre que la frise, est blanche, ce qui est invraisemblable (fig. 4 et 5). Cette décoration, oil le blane domine, est grêle ct sèche; on la rencontre sur certaines plinthes refaites de la seconde salle, et dans une autre maison de Glanum, dont un vestige de mur a été découvert en 1964 entre les "temples géminés".

\section{Peinture sous le péribole des temples géminés (entre XXIII et XXIV).}

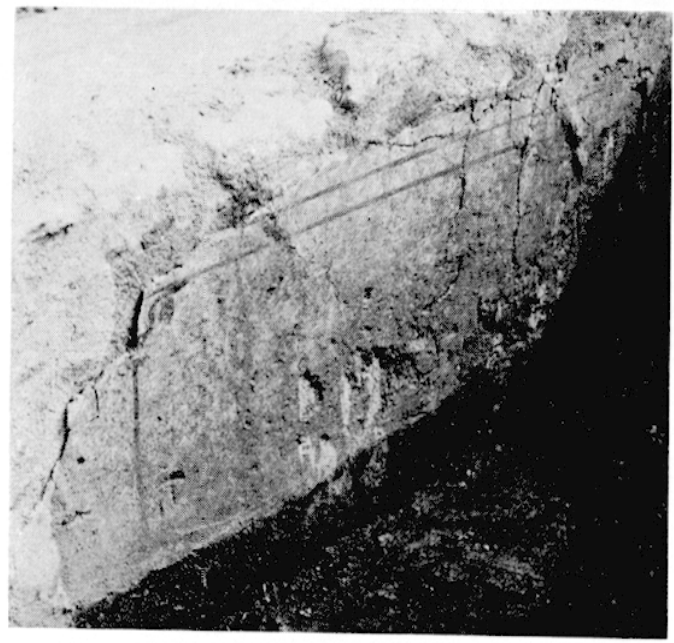

7 Gianum, mur sous le péribole des temples géminés (XXIII et XXIV).

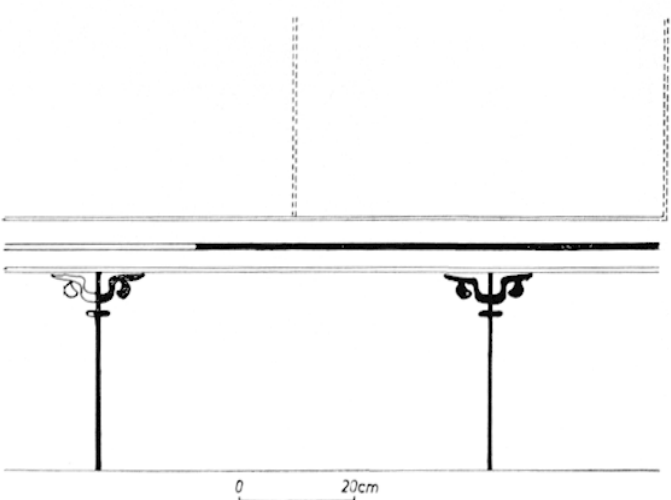

8 Glanum, mur sous le péribole des temples géminés (XXIII et XXIV).

Ce vestige de mur, situé sous le niveau de Glanum III auquel appartiennent les deux temples, est de la même époque que la maison aux deux alcôves (XVIII). Il porte un décor de deux hampes noires, éloignées de $68 \mathrm{~cm}$ l'une de l'autre et qui s'enlève sur un fond blanc. Les hampes sont peu hautes $(30,5 \mathrm{~cm})$ et peu épaisses $(0,7 \mathrm{~cm})$, elles sont surmontées de deux volutes d'un dessin un peu différent, précédées d'une barre horizontale. Au-dessus de cette plinthe, trois filets horizontaux et parallèles : un noir compris entre deux rouges (fig. 7 et 8). C'est le même jeu qui se remarque sur les murs de la première salle de la maison aux deux alcôves (XVIII), avec des différences sensibles dans les proportions et la forme des volutes. Il faut conclure qu'une même équipe de décorateurs a travaillé dans ces deux maisons de Glanum, exécutant un décor simplifié et parfois même déformé.

Toutes les erreurs et les maladresses d'exécution, surtout dans les parties hautes du décor de la maison aux deux alcôves (XVIII), font deviner un modèle romain, dérivé du Second style. Or ce motif de hampes et de volutes se rapproche de façon frappante de toute une série de documents, présentant la même caractéristique d'un fond blanc et, en général, d'un jeu unique de couleurs rouge et noire, avec de nombreuses variantes dans les volutes. L'aire de répartition de ce style pictural est intéressante, nous l'appellerons "Second style schématique ". Un foyer est reconnaissable en Grèce, à Théra, Athènes et 


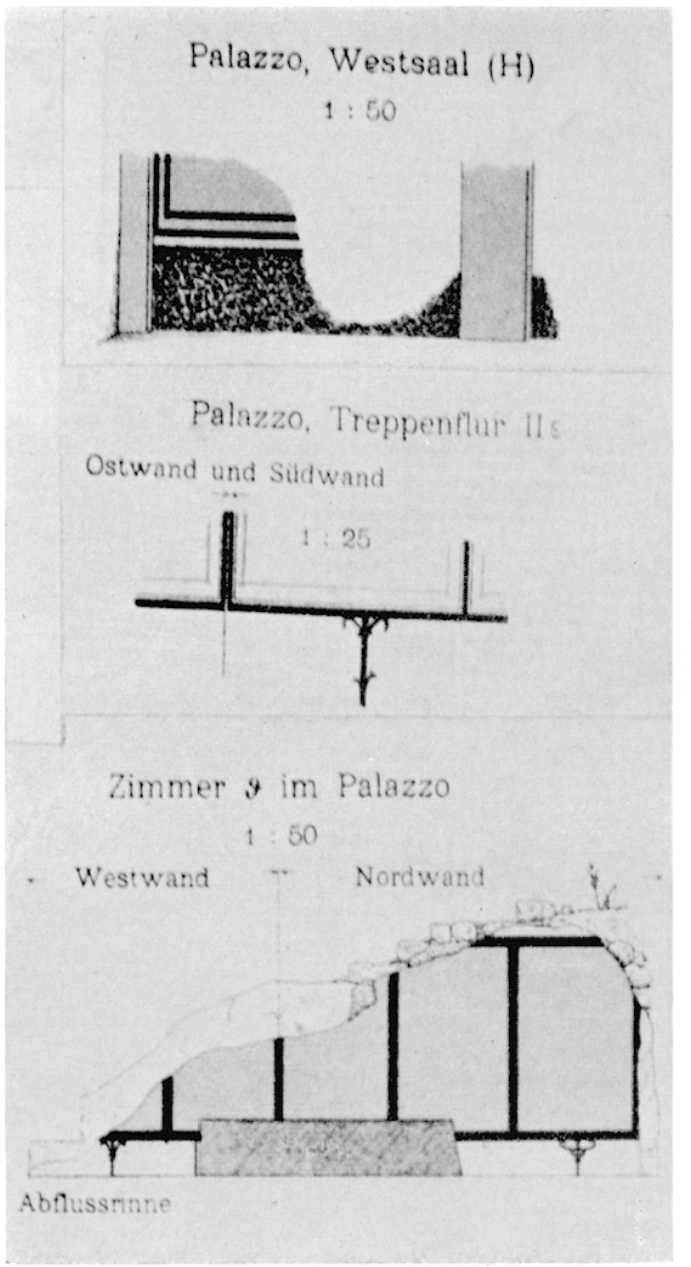

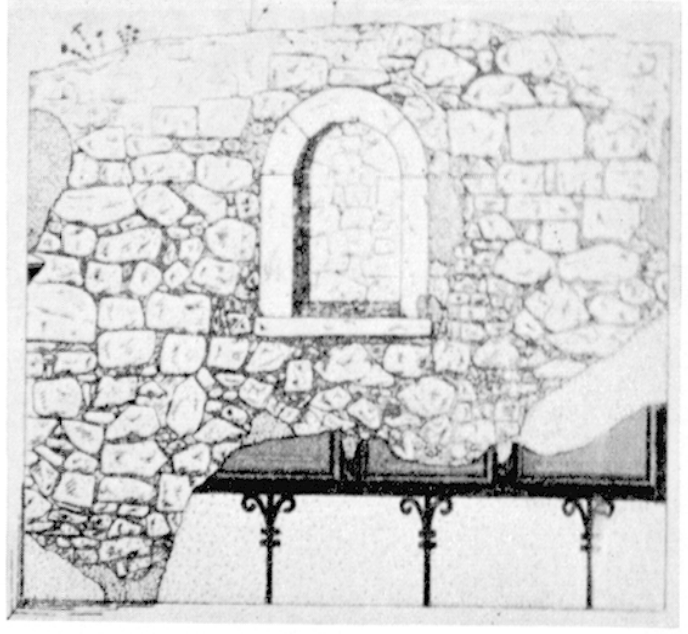

11 Thera, maison I).

«- 10 Théra, Jo palais, salle H, escalier II $\varepsilon$, chambre: 0 .

murales qui semblent, d'après l'auteur, former un ensemble ${ }^{5}$. Les motifs de plinthe de ces salles accusent deux types de hampes à volutes:

a) hampes à volutes proches de celles de Glanum, avec fleuron central, par exemple sur le mur ouest de la salle $\Theta$ (fig. 10), accompagnées de deux traits sous les volutes. De même, dans le corridor de l'escalier II $\varepsilon$, où les volutes ont l'allure de plante, surtout à cause de deux petits motifs semblables à des feuilles qui viennent s'attacher sur la hampe. Même type, aussi, sur le mur nord de la salle $\mathrm{S}$, où deux hampes (celles des extrémités) sont soulignées de trois petites barbes (fig. 12);

b) le second type ressemble tout à fait à un caducèe. Les deux volutes, après s'être croisées comme des serpents sur la hampe, sont recourbées vers l'intérieur, par exemple sur le mur nord de la salle $\Theta$ (fig. 10); il en est de même pour la volute centrale de la salle $S$ (fig. 12).

Cependant les plinthes sont rarement d'un blanc uni, elles sont blanches et mouchetées de rose pour la salle $\Theta$. Les orthostates sont plus colorés, plus ornés qu'à Glanum. Dans le corridor de l'escalier II $\varepsilon$ ils sont dessinés à l'aide de traits noirs, ocre jaune et rouge, dans la salle $\mathrm{S}$ les orthos-

(5) Thera, t. III, par Hiller vox Gaertrixgex (Berlin, 1905), pl. 1 à 3, p. 157, 163 à 169. 


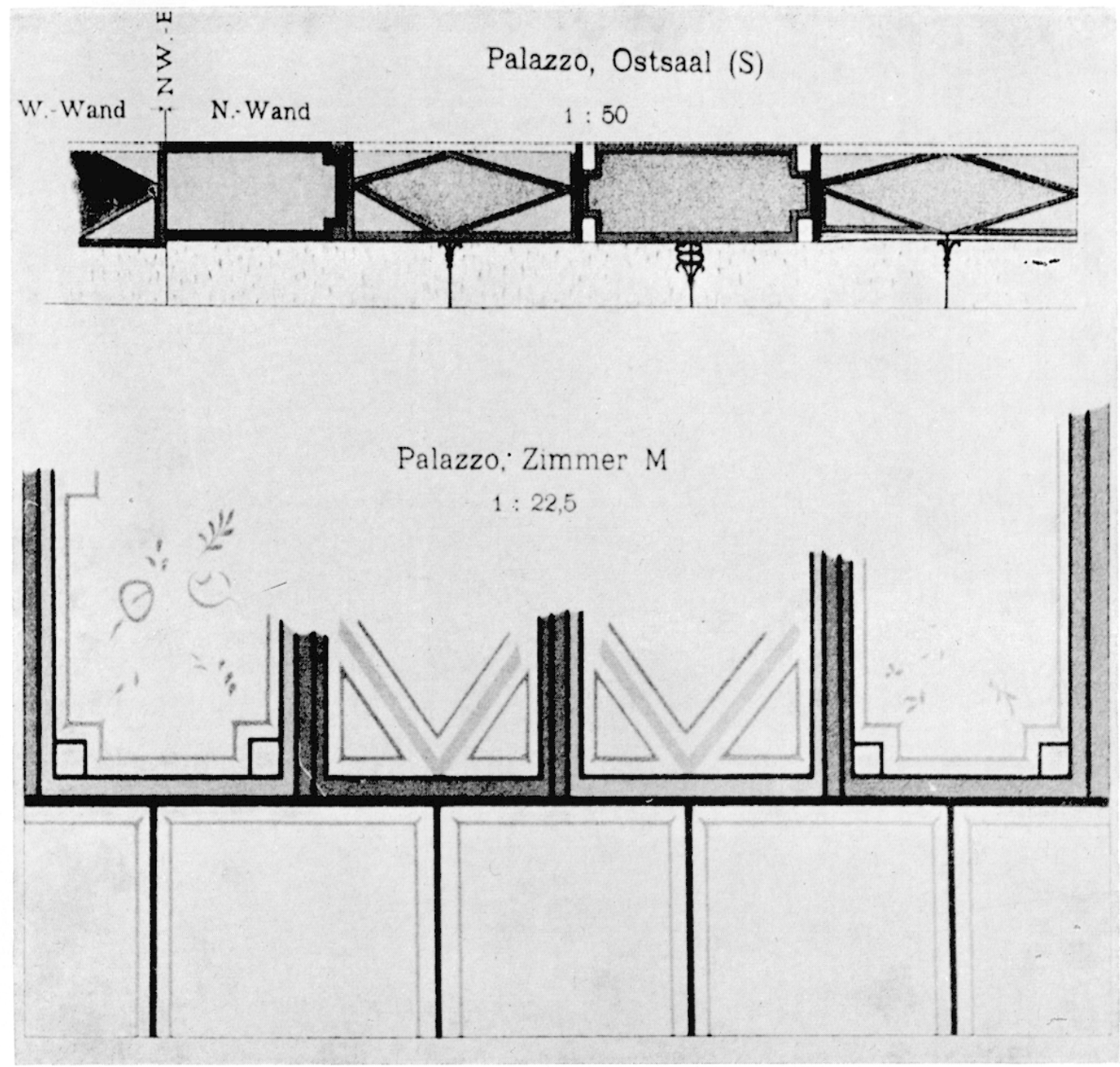

12 Théra, le palais, salle $\mathrm{S}$ et chambre $\mathrm{M}$.

tates de couleurs vives sont en forme de losanges couchés alternant avec des plaques rectangulaires entourant des cartouches (fig. 12). La pièce $\mathrm{H}$ contient un décor différent.

La pièce $H$ ou salle de l'ouest: une plinthe basse, sombre, mouchetée de noir, est scandée par des piliers roses réalisés en stucs en relief. La datation proposée par Hiller von Gaertringen pour cette salle oscille entre le $\mathrm{II}^{\mathrm{e}}$ siècle av. J.-C. et le $\mathrm{II}^{\mathrm{e}}$ siècle ap. J.-C. Il pense que si les peintures des deux salles de l'ouest [salle $H$, escalier $I I \varepsilon$ ] appartiennent au II $^{\mathrm{e}}$ siècle av. J.-C., ce serait au moment du renouveau du gymnase et du départ des garnisons de Ptolémée. Des particuliers occupèrent les bâtiments, changèrent la disposition des pièces, qui furent peintes. Ces peintures subistèrent jusqu'à l'époque impériale ; si, au contraire, les peintures sont de l'époque impériale, elles seraient du ${ }_{{ }^{\mathrm{I}}}{ }^{\mathrm{e}}$ siècle ap. J.-G., époque de Clitosthène. L'auteur affirme que, vue l'unité des divers bâtiments de la même époque, il faut pencher pour la première ou pour la seconde solution. L'alternative qu'il pose montre son hésitation à placer ces décors dans la peinture grecque ou dans la peinture romaine. 
Le plan d'ensemble des fouilles ${ }^{6}$, prouve que les bâtiments ont été édifiés sans ordre, au gré des époques successives et que "l'unité » des constructions, affirmée par Gaertringen est douteuse. Les salles de l'ouest (H et II $\varepsilon$ ) et de l'est (S) existaient au temps du gymnase et furent par la suite englobées dans toute une série de bâtiments.

D'autres maisons situées à l'ouest du portique de la basilique contenaient des décors à hampes à volutes.

2. La maison D. -.. Dans la maison D, par exemple, la plinthe de la chambre D 3 (mur sud) est claire, assez basse, avec hampes à volutes soulignées de deux traits, tout à fait semblable à celles du Palais (chambre $\Theta$ et $S$ ). Elle est surmontée d'orthostates séparés les uns des autres par d'étroites bandes noires ornées de motifs incomplets et difficiles à interpréter (motifs floraux ?) (fig. 11).

La parenté est évidente entre les décors de ces deux édifices, le palais et la maison D. On y remarque une même organisation : une plinthe avec hampes à volutes surmontées d'orthostates rectangulaires ou losangiques, avec préférence pour les fonds clairs.

Nous avons vu qu'il fallait mettre à part le décor de la salle $\mathrm{H}$ du palais, avec piliers en stuc en relief, qui tranche sur les autres décors et que von Gaertringen propose à son sujet soit le $\mathrm{II}^{\mathrm{e}}$ siècle av. J.-C., soit le II ${ }^{\mathrm{e}}$ siècle ap. J.-C. ${ }^{7}$. Or, en ce qui la concerne, il n'y a aucun doute : il s'agit d'un décor typique de la peinture murale grecque de la fin du II $^{\mathrm{e}}$ siècle av. J.-C., telle que nous la trouvons à Délos; elle fut imitée par les Romains jusque dans les premières années du I ${ }^{\text {er }}$ siècle av. J.-C., où elle donna le Premier style de Pompéi, défini par A. Mau. Il s'agit donc soit d'un décor grec de la fin du II $^{\mathrm{e}}$ siècle av. J.-C., soit d'un décor romain, qui peut s'étendre aux premières années du ier siècle av. J.-C. Le peu qui en reste ne permet pas de choisir l'une ou l'autre solution.

Le plan du palais montre que les deux salles qui existaient à l'époque du gymnase grec sont les salles $\mathrm{H}$ et $\mathrm{S}$. Le décor de la salle $\mathrm{H}$, s'il est incontestablement ancien et peutêtre grec, ne prouve évidemment pas que le décor de losanges et de hampes à volutes de la salle S soit de la même époque. Le problème se pose de savoir si les décors autres que celui de la salle $H$ sont homogènes. Il semble qu'il faille dissocier les peintures que nous venons d'analyser de celles de la salle. $\mathrm{H}$, nettement romaines, à notre sens (fig. 12). Par exemple, le décor floral léger des plaques rectangulaires de la salle $M$ ne se rencontre nulle part dans la peinture murale grecque de style structural, tels que les documents qui nous sont parvenus le démontrent. L'hypothèse d'une unité des peintures correspondant à une unité des corps de bâtiments est donc faussc et reconnue comme hasardeuse par l'auteur même ${ }^{8}$.

Il existe à Théra des peintures de style structural grec, dans la maison de Pothétos par exemple, où fut retrouvée une tresse avec bossage en relief vert ${ }^{9}$. Dans cette mème

(6) Thera, op. cit., fig. 136 et 139 .

(7) Thera, op. cit., p. 169.

8) En effet, von Gaertringen reconnaît que les peintures de la salle $M$ doivent être plus " jeunes " que les autres.

(9) Elle devrait être placée horizontalement et non pas verticalement (pl. 4 de la publication de Théra), comme le fait remarquer M. Bulard, Peinlures murales el mosaiques de Délos, dans Monuments et mémoires de la fondation E. Piof, XIV, 1908, p. 121, n. 1 . 
maison, une pièce était décorée d'une plinthe claire avec motifs de tridents à volutes de même inspiration que les autres (fig. 15 a). Ce type de décor est donc bien en corrélation avec le Premier style grec, qui date au plus tôt des premières années du i ${ }^{\text {er }}$ siècle av. J.-C. Ce fait suggère une datation précoce du type de pcinture à hampes et à volutes.

Il résulte des observations de Hiller von Gaertringen que les peintures de Théra auraient été exécutées à deux époques, séparées par plus de trois siècles d'intervalle :

a) la première époque: les peintures de style structural, de la fin du ${ }_{{ }^{2}}{ }^{\mathrm{e}}$ siècle av. J.-C. ou du début du $\mathrm{I}^{\mathrm{er}}$ siècle (c'est-à-dire grecques ou romaines) sont représentées par le fragment de tresses et de bossages en relief de la maison de Pothétos et par les piliers en stucs en relief de la salle $\mathrm{H}$ du palais.

b) la seconde époque: caractérisée par des peintures à fond blanc, à hampes et à volutes noires, elle est abondamment représentée, dans le palais, dans la maison $\mathrm{D}$ et celle de Pothétos (avec tridents).

Il nous parait inconcevable qu'un tel intervalle de trois siècles puisse être envisagé entre ces deux types de décor, que l'on rencontre ensemble dans une même maison (celle de Pothétos) et dans deux salles (le premier type dans la salle $\mathrm{H}$, le second dans la salle $\mathrm{S}$ ) édifiées ensemble, contemporaines du gymnase grec, noyau autour duquel fut construit le palais. A notre sens, il faut admettre un maximum d'intervalle d'un siècle entre ces deux types de décor, comme les comparaisons établies avec des documents analogues nous le montreront; ils sont tous à dater de la seconde moitié du $\mathrm{I}^{\mathrm{er}}$ siècle av. J.-C. Nous rejoignons par là le jugement de $\mathrm{A}$. Conze et de $\mathrm{C}$. Robert, qui datent les peintures du palais de Théra du II $^{\mathrm{e}}$ et du I ${ }^{\mathrm{er}}$ siècle av. J.-C. ${ }^{10}$

\section{Délos.}

A Délos, sur l'emplacement de la salle hypostyle, furent construites des maisons, dont une $\left(\mathrm{n}^{0} \mathrm{~V}\right)$ est décorée de peintures ${ }^{11}$. Dans la chambre $m$, elles forment des motifs linéaires et végétaux d'une grande simplicité, se détachant sur un fond blanc uni. A hauteur d'appui, une bande horizontale partage la surface de chaque mur en deux zones:

la zone supérieure est divisée verticalement en rectangles ou en carrés par des bandes rouges doubles, entre lesquelles se dresse une mince tige rouge, avec feuilles et fleurs. A l'intérieur de chaque compartiment, un filet jaune ou rouge dessine un encadrement (fig. 13) ;

la zone inférieure est scandée, sous le milieu de chaque compartiment, par des hampes, ornées à leur partie supérieure de deux traits en croix ou de plusieurs (sept dans un cas), formant barbelures. Elles s'épanouissent en deux volutes, doubles pour chaque côté, à allure de rinceaux et portant des tridents. De ses trois pointes, chaque trident touche et semble soutenir le cadre des compartiments supérieurs (fig. 14).

D'après G. Leroux, la maison $\mathrm{n}^{0} \mathrm{~V}$ de Délos est romaine et de préférence de l'époque antonine ${ }^{12}$; elle fut construite - comme les autres ( $n^{08}$ I à IV) - après la destruction de la salle hypostyle, élevée à la fin du me siècle av. J.-C. et qui dut subsister jusqu'aux approches

(10) Cité dans Thera, III, p. 169.

(11) G. Leroux, Exploralion archéologique de Délos, II, 1909, p. 64 et s.

(12) Ibid, p. 71-72. 


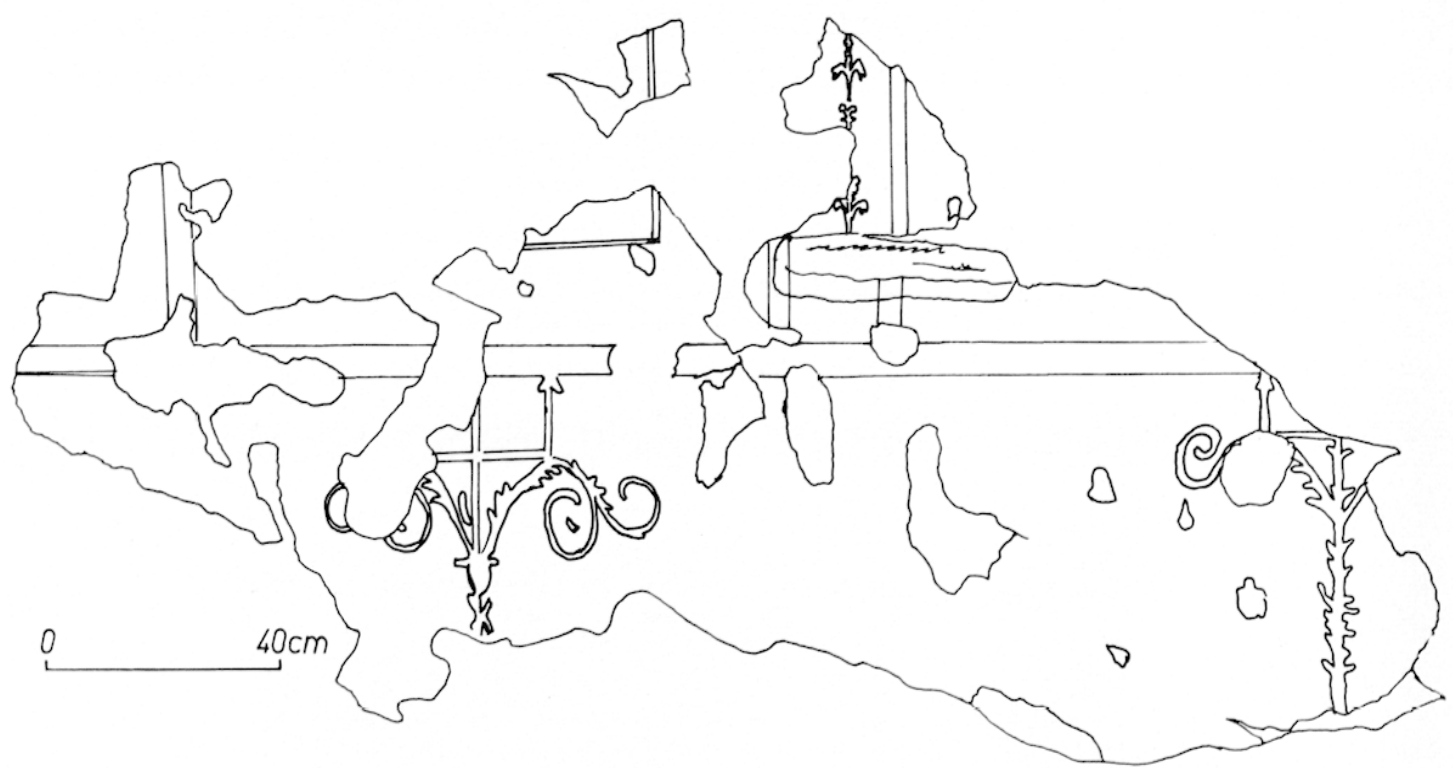

13 Délos, maison romaine $\mathrm{n}^{\circ} \mathrm{V}$, chambre $m$, mur est.

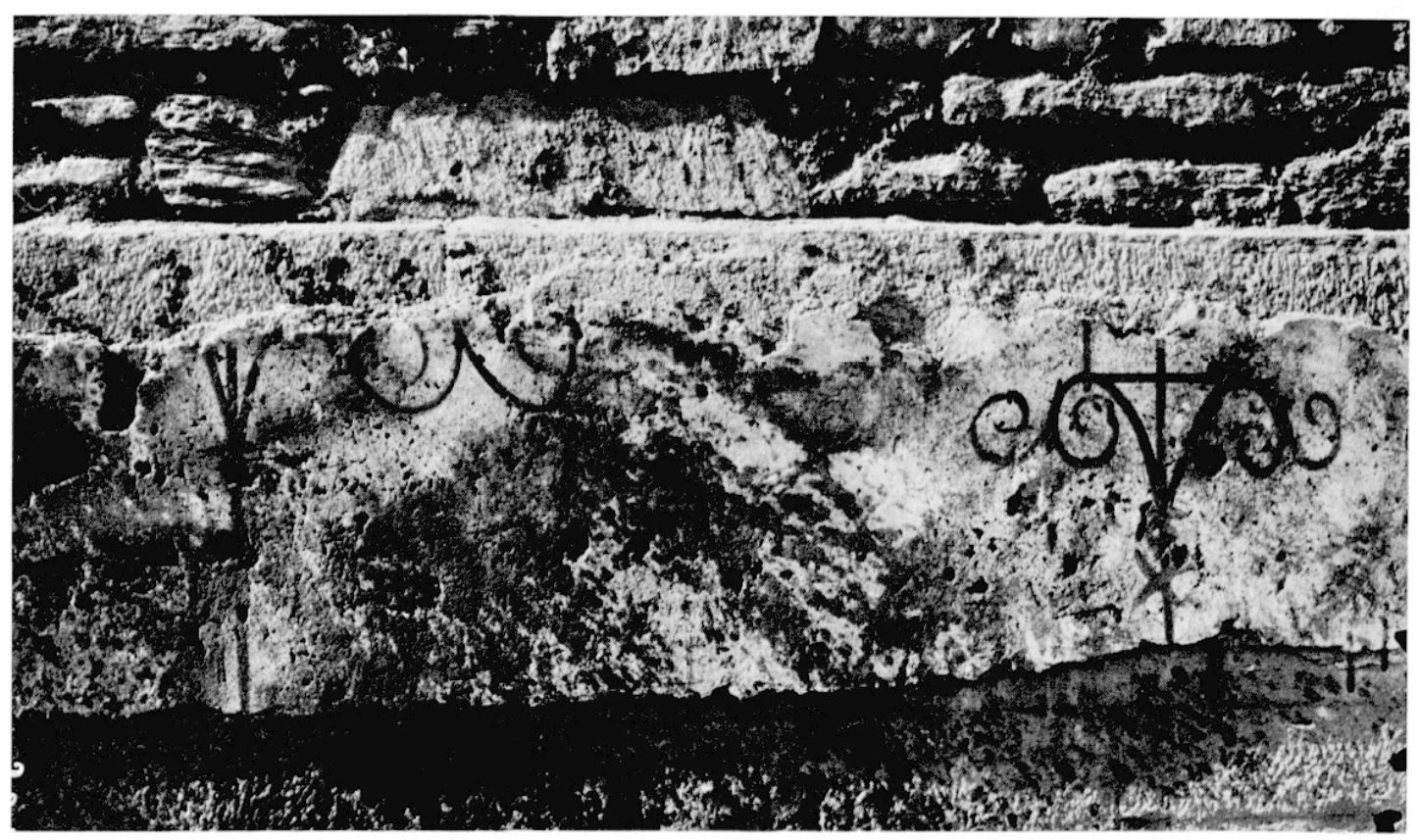

14 Délos, maison romaine $n^{\circ} \mathrm{V}$, chambre $m$.

de notre ère. Ciette maison est plus ancienne, évidemment, que les constructions byzantines qui l'ont recouverte. On y a mis au jour des lampes de type romain du $\mathrm{I}^{\mathrm{er}}$ siècle ap. J.-C. Comparant ce décor avec celui de Théra, influencé par la datation antonine de Hiller von Gaertringen, l'auteur date ces peintures de Délos de l'époque impériale, à un âge plus récent que les derniers styles pompéiens, dont elles marqueraient l'appauvrissement et l'aboutissement. Lne autre maison de Délos possède un décor semblable; édifiée au Ive siècle av. J.-C., elle n'a reçu son décor qu'à une époque romaine avancée. 
Les indices de datation exposés par G. Leroux pour ces deux maisons ne sauraient tous être retenus, notamment les critères stylistiques très fragiles, fondés sur les analyses de Hiller von Gaertringen à Théra, que nous avons précédemment examinées. Bien plus, la présence de lampes romaines du $\mathrm{I}^{\text {er }}$ siècle ap. J.-C. dans la maison V de Délos ne raffermit pas l'hypothèse d'un décor de l'époque antonine mais, au contraire, l'affaiblit. La trouvaille de ces lampes, considérées comme le matériel le plus récent, indique uniquement que l'habitation était utilisée au $\mathrm{I}^{\mathrm{er}}$ siècle ap. J.-C., sans que l'on puisse préjuger de son décor qui peut être antérieur ou contemporain. On peut même conclure que ces lampes, derniers témoins du mobilier de la maison, attestent la dernière période d'occupation de cette demeure et que par conséquent les peintures ne sont pas postérieures à cette date.

D'après Ph. Bruneau ${ }^{13}$, l'examen des lampes romaines, en dépit de leur petit nombre, permet de faire les conclusions suivantes : l'île a été occupée sans interruption jusqu'au vi ${ }^{\mathrm{e}}$ siècle ap. J.-C., au moins, sans abandon au i ${ }^{\mathrm{er}}$ siècle. La lampe $\mathrm{n}^{\circ} 4577$, trouvée dans une des maisons édifiées à l'emplacement de la salle hypostyle, qui ont èté considérées par G. Leroux comme étant du II $^{\mathrm{e}}$ siècle ap. J.-C., est sûrement attribuable au $\mathrm{I}^{\mathrm{er}}$ siècle ap. J.-C., et permet de rajeunir ces édifices. Ce type de lampe, d'origine italienne, s'est répandu dans tout le monde méditerranéen de la fin du $\mathrm{I}^{\mathrm{er}}$ siècle av. J.-C., jusque dans le courant du $\mathrm{II}^{\mathrm{e}}$ siècle ap. J.-C. Les exemplaires les plus anciens, remontant au règne d'Auguste, sont caractérisés par une forme de disque et de volutes, qui évolue ultérieurement ${ }^{14}$. La lampe 4577 paraît très proche des débuts de cette série. Rien ne s'oppose donc à faire remonter ces décors à la fin du ${ }^{\mathrm{er}}$ siècle av. J.-C., lorsque la maison fut bâtie sur les ruines de la salle hypostyle.

\section{Alhènes.}

Deux maisons d'époque romaine, établies entre l'Aréopage et la Pnyx, renfermaient des peintures analogues à celles de la maison $\mathrm{V}$ de Délos ${ }^{15}$. L'une d'elles présente une plinthe blanche surmontée de deux volutes, avec pointe centrale terminée en flèche. Au-dessus, s'étendent des compartiments rectangulaires limités par des filets; ils sont séparés par des bandes verticales occupées par des motifs végétaux. L'auteur ne donne pas d'autres précisions sur la date de cet édifice (fig. 15 b).

\section{Ensérune.}

Sur cet oppidum, situé à quclques kilomètres de Bćzicrs (Hćrault), des maisons d'époque romaine ont livré deux sortes de décor :

le premier est à fond blanc, sur lequel se détachent en rouge et en noir des filets d'encadrement, qui le divisent en compartiments ornés de divers motifs décoratifs. Par exemple, les compartiments peuvent être ornés de grosses pastilles rouges et noires, de tiges à volutes figurant des sortes de

(13) P. Bruneau, Les lampes, dans Exploration archéologique de Délos, 26, 1965, p. 14, n. 3.

(14) P. Bruneau, op. cit., p. 115.

(15) G. Leroux, op. cit., fig. 101-102, p. 71. 

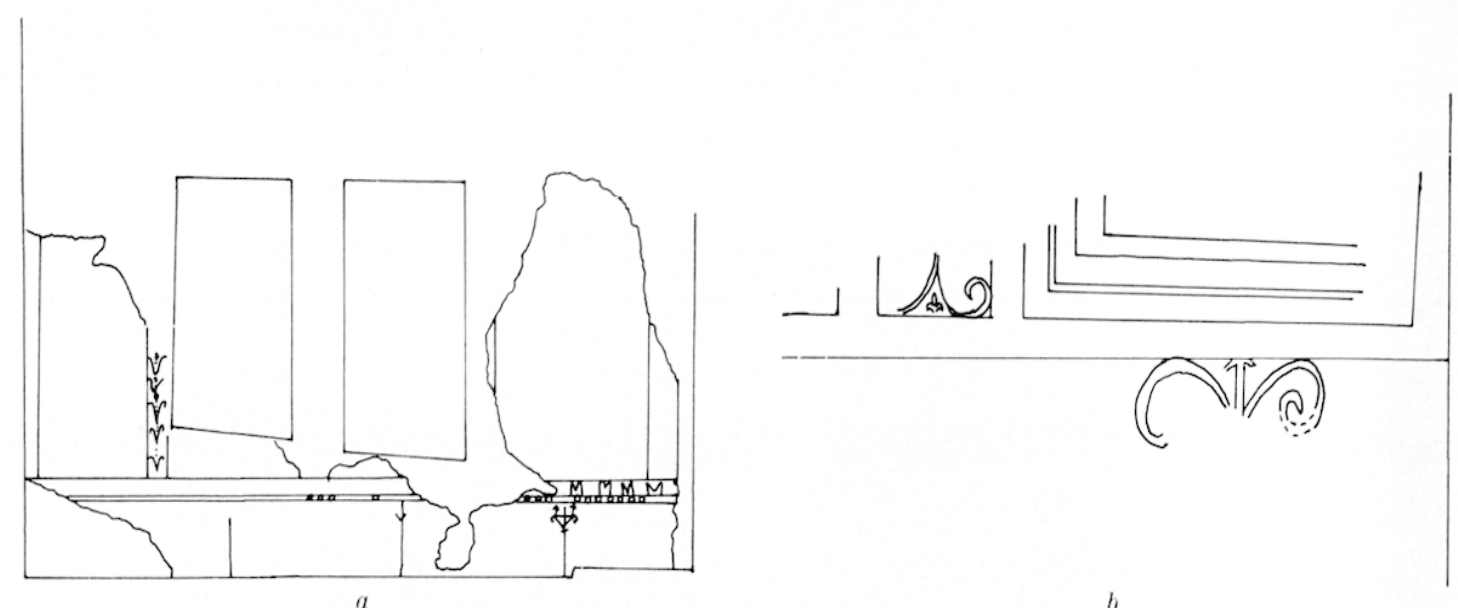

15 a Théra, maison de Pothétos, salle $G ; b$ maison romaine d'Athènes.

thyrses peints en noir, de dessins variés traités en rouge, cercles concentriques réunis par des zigzags et des perles ou plantes foliacées ${ }^{16}$;

le second groupe, avec une gamme de couleurs étendue, se caractérise par une ornementation guillochée ou jaspée, évoquant des plaques de marbres; il appartient au Second style pompéien.

Le premier groupe seul nous retiendra pour la comparaison avec Glanum et les sites grecs étudiés précédemment. Il nous parait plus ancien que le second, alors que J. Jannoray les considérait comme contemporains. Le premier argument de l'auteur ${ }^{17}$ est leur découverte dans des édifices qui se sont effondrés au même moment. Cela ne prouve pas que les peintures des deux manières étaient contemporaines ; une maison a pu subir des réfections et présenter deux types d'enduits peints. Le second argument est le suivant : dans la chambre III, 16, un décor de la première manière est conservé sous un décor de la seconde. J. Jannoray en déduit que les deux procédés ont été utilisés en même temps, alors que, croyons-nous, cette découverte impose de facon certaine la réalité de deux phases successives, plus ou moins éloignées dans le temps.

Maison aux pseudo-thyrses (II, 7). Dans l'insula II, pièce 7, le revêtement était demeuré en place dans l'angle s.-e. et au centre de la paroi, sur une hauteur de $0,60 \mathrm{~m}$ à partir du niveau marqué par le pavement. On y a recueilli de nombreux débris des superstructures gardant encore leur enduit ${ }^{18}$. Il a été possible de tenter une restauration d'ensemble. Celleci montre que la décoration de la salle comportait trois registres superposés (fig. 16) :

une plinthe à fond blanc, constituée par une série de compartiments d'une largeur moyenne de $83 \mathrm{~cm}$ pour une hauteur de $60 \mathrm{~cm}$ que délimitaient des "thyrses " peints en noir ;

une zone supérieure d'orthostates blanes, séparés de la plinthe par un filet horizontal, constitués de traits noirs, que doublait intérieurement un second trait, noir en haut et à droite, rouge en bas et à gauche ;

(16) J. Jannoray, Ensérune, contribution à l'élude des civilisations pré-romaines de la Gaule méridionale, Paris, 1955, p. 111-112.

(17) J. JANNORAY, op. cit., p. 118.

(18) Ibid., p. 114. 

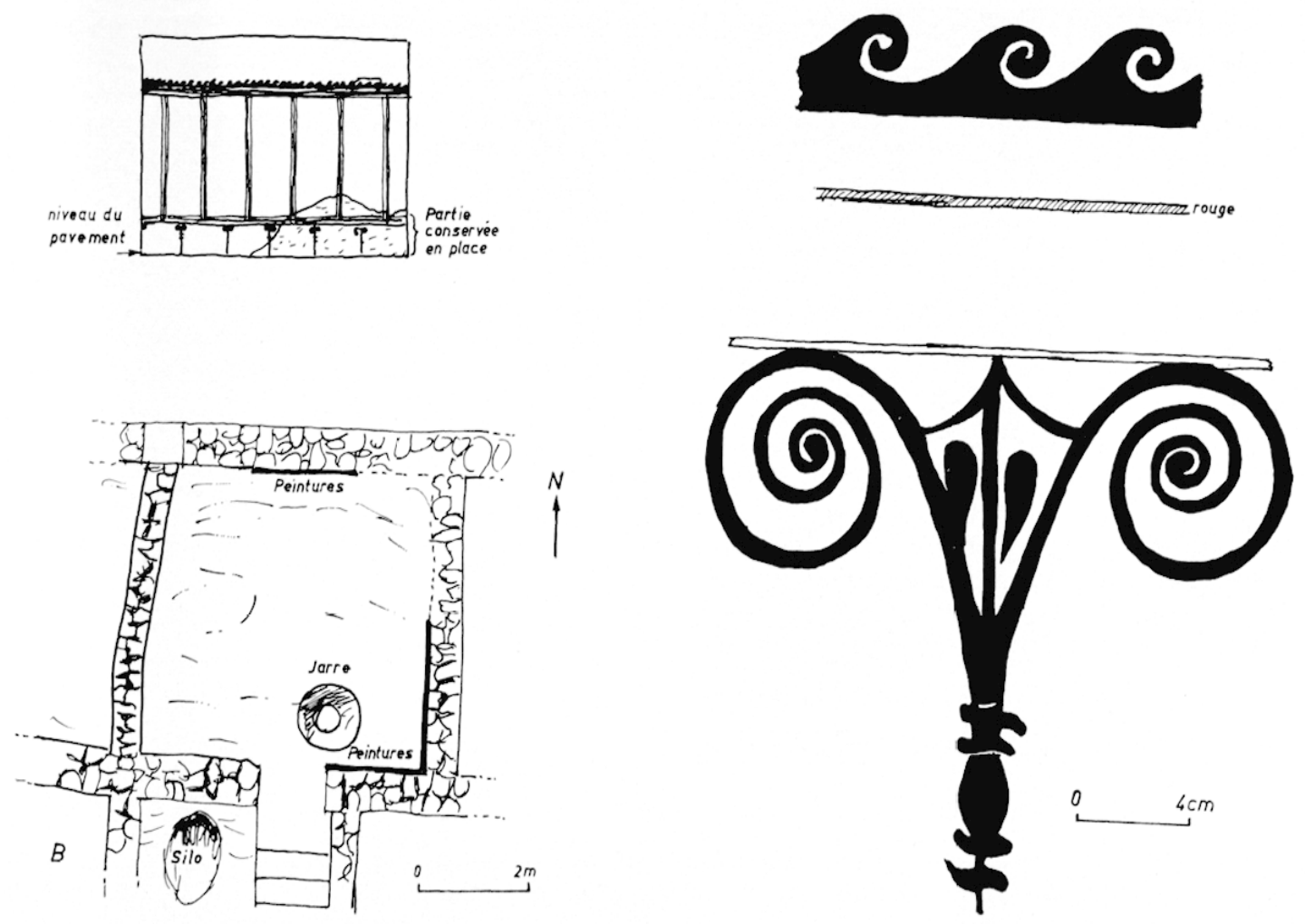

16 Ensérune, maison aux pseudo-thyrses (Ins. 1I, 7), d'après Jannoray.
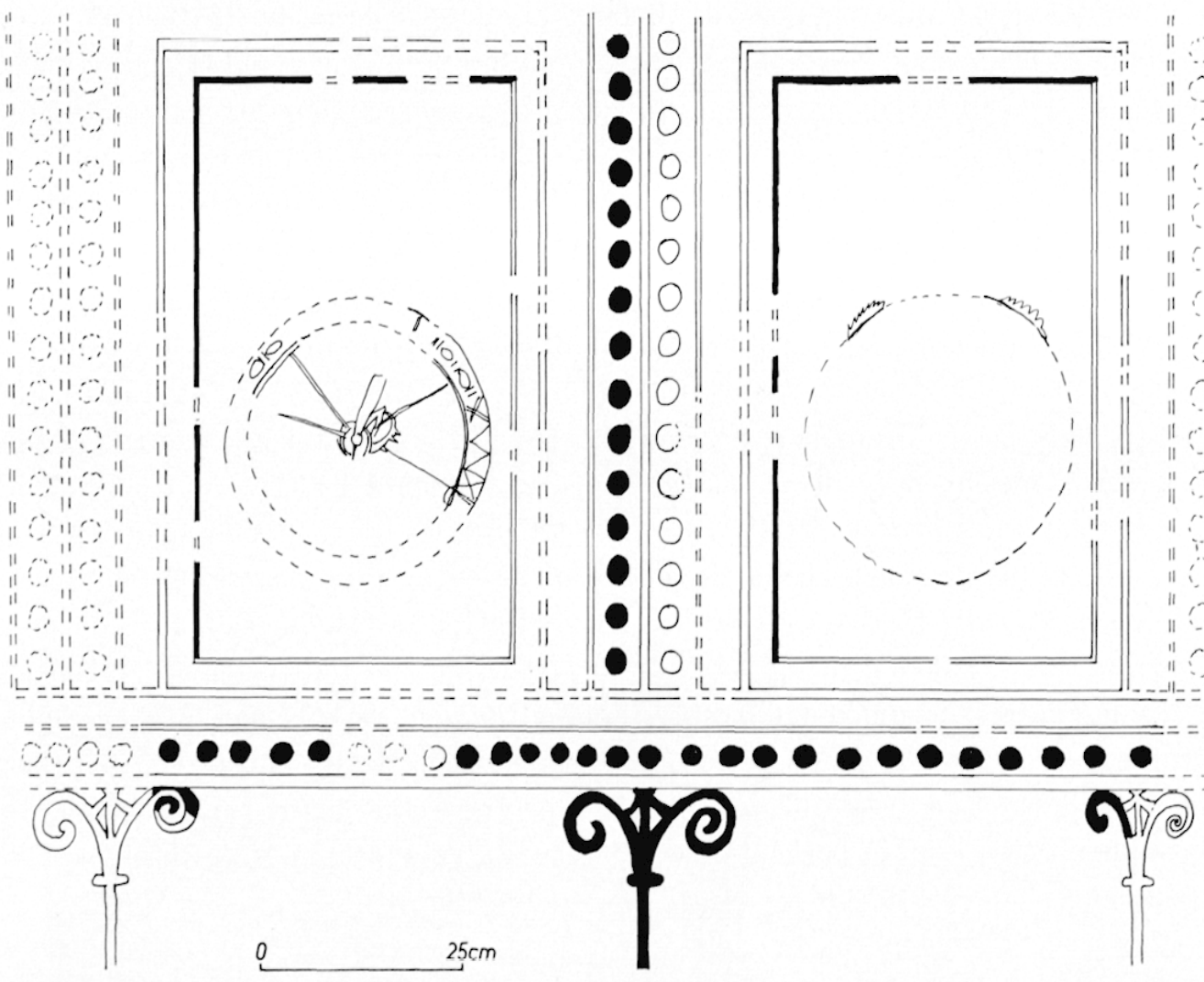

17 Ensérune, maison aux pseudo-thyrses (Ins. II, 7), relevé à partir de la reconstitution de l'abbé Sigal. 


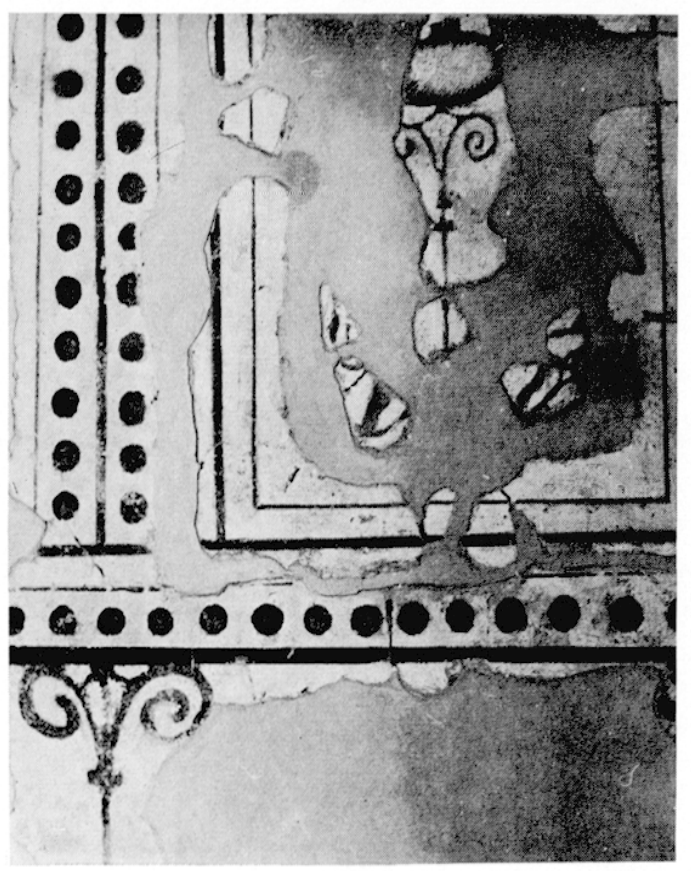

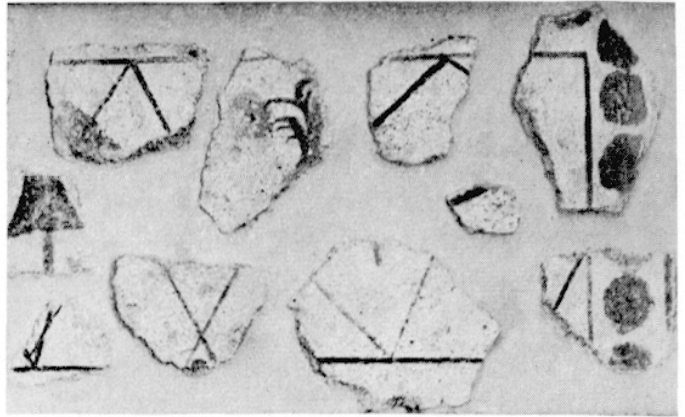

19 Ensérune, maison aux pseudo-thyrses (Ins. II, 7), fragments d'enduit dans leur état originel.

«- 18 Ensérune, maison aux pseudo-thyrses (Ins. II, 7), partie droite du panneau reconstitué par l'abbé Sigal.

une frise de postes formait couronnement au sommet de ces quadrilatères. Les "thyrses " ressemblent de très près aux volutes de la première salle de la maison aux deux alcòves (XVIII) de Glanum. Même dessin mais plus fin, plus détaillé, même fond blanc uniforme aussi bien pour la plinthe que pour les orthostates, tracés au moyen d'un jeu de lignes rouges et noires.

En se fondant sur le matériel recueilli, J. Jannoray date ce décor du ier siècle av. J.-C. En effel, le mobilier mis au jour indique une occupation durant la seconde moitié de ce siècle. On a trouvé de la poterie arétine, peu de céramique catalane, très rarement du campanien. Une monnaie datée de 89 av. J.-C. a été découverte. La maison a donc été habitée au plus tard dans la seconde moitié du I $^{\text {er }}$ siècle av. J.-C. Le décor appartient vraisemblablement à cette époque, mais il peut être un peu plus ancien.

Lorsqu'on compare la restitution graphique proposée par J. Jannoray et les panneaux exposés au musée d'Ensérune, des différences notables sautent aux yeux. Le panneau (130 de long $\times 105,5 \mathrm{~cm}$ de haut) reconstitué au musée par l'abbé Sigal fait apparaitre des restaurations abusives. Un examen approfondi montre que la surface blanche originelle de l'enduit a été badigeonnée de blanc, partant très facilement à l'eau, ce qui permet de retrouver dessous le dessin réel (fig. 17, 18). Tout d'abord, les fragments de peinture avec les volutes ont été placés sous les traits de séparation des orthostates, le restaurateur ayant masqué les raccords entre les cassures qui ne coïncidaient pas ; J. Jannoray, au contraire, les fait figurer sous le milieu de chaque orthostate, conformément au schéma généralement adopté sur les autres documents de cette série, à Glanum et à Rome, par exemple. Les pastilles rouges et noires disposées autour des orthostates déteignent au lavage; elles sont donc régularisées ou créées de toutes pièces par le restaurateur, sur le modèle de pastillages plus petits, ceux-là authentiques, conservés à l'état fragmentaire au musée et qui ne figurent pas dans le dessin donné par J. Jannoray (fig. 19). 

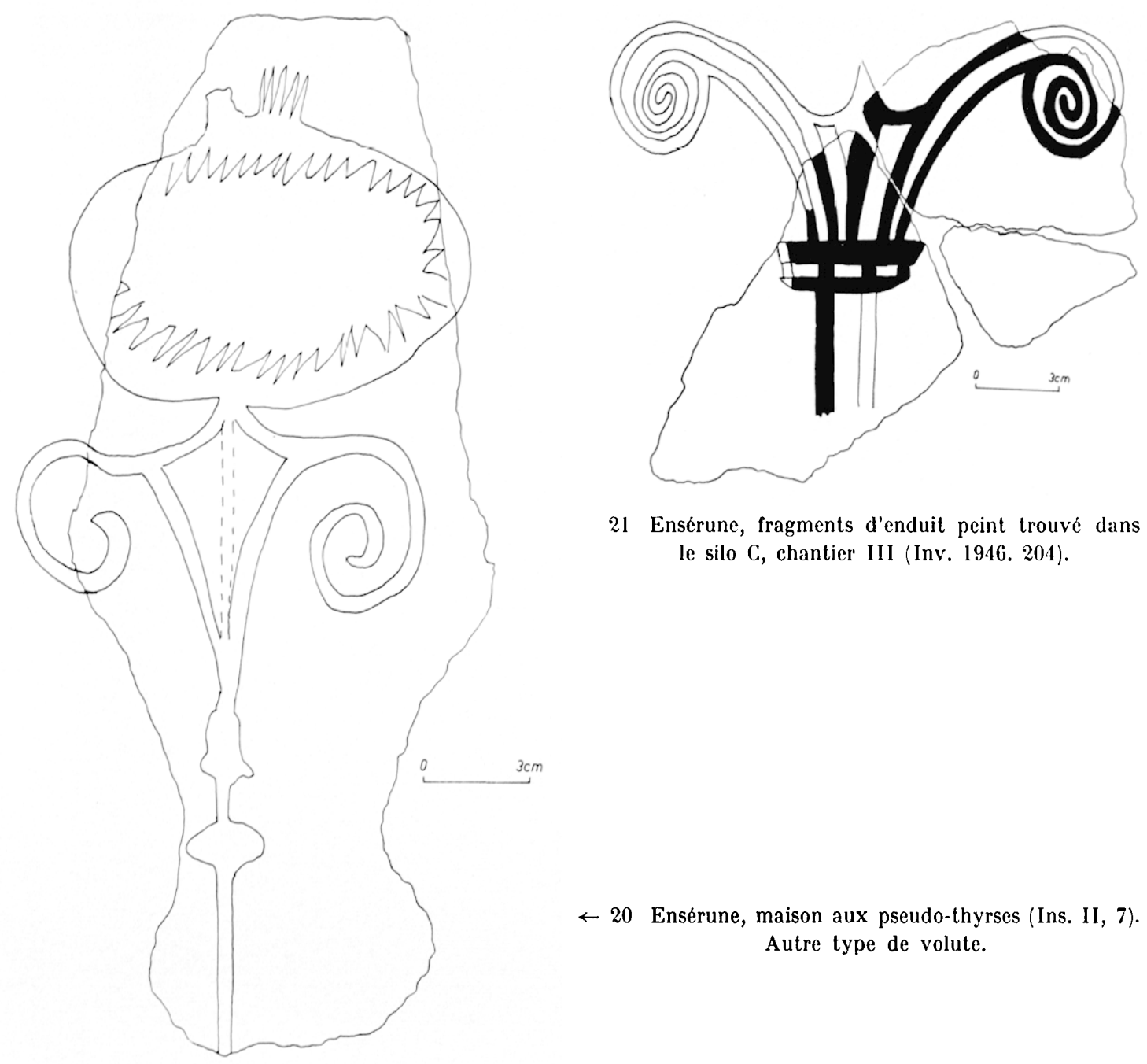

21 Ensérune, fragments d'enduit peint trouvé dans le silo C, chantier III (Inv. 1946. 204).

$\leftarrow 20$ Ensérune, maison aux pseudo-thyrses (Ins. II, 7). Autre type de volute.

Enfin, ce qui est important, les volutes présentées au musée ne sont pas semblables à celle qui est reproduite par J. Jannoray. Elles sont moins enroulées et les deux gouttes du bulbe n'existent pas. Un lavage soigneux de cette partie ne révèle aucune goutte pour la volute centrale du panneau restauré par l'abbé Sigal. Faut-il en conclure que l'exemplaire avec gouttes donné par J. Jannoray a disparu ou que son dessin est inexact ? Le cas est obscur car le dessinateur de l'exemplaire fourni par J. Jannoray est justement l'abbé Sigal, dont la signature est bien visible.

Au centre du panneau supérieur de droite a été placée, lors de la reconstitution, une plaque d'enduit à fond blanc décorée d'une tige et de deux volutes peintes en ocre rouge et surmontées d'un grand ovale avec toupet hérissé à la partie supérieure. La hampe présente deux renflements latéraux (fig. 20). On ne sait si ce nouveau type de volute a été trouvé dans la même maison; il semble seulement exclu qu'il provienne de la même pièce. Son emplacement devait se situer probablement sur une plinthe.

L'examen des caisses de peinture murale du musée d'Ensérune nous a permis de découvrir un troisième type de volute pour ce seul habitat. Les trois fragments d'enduit 


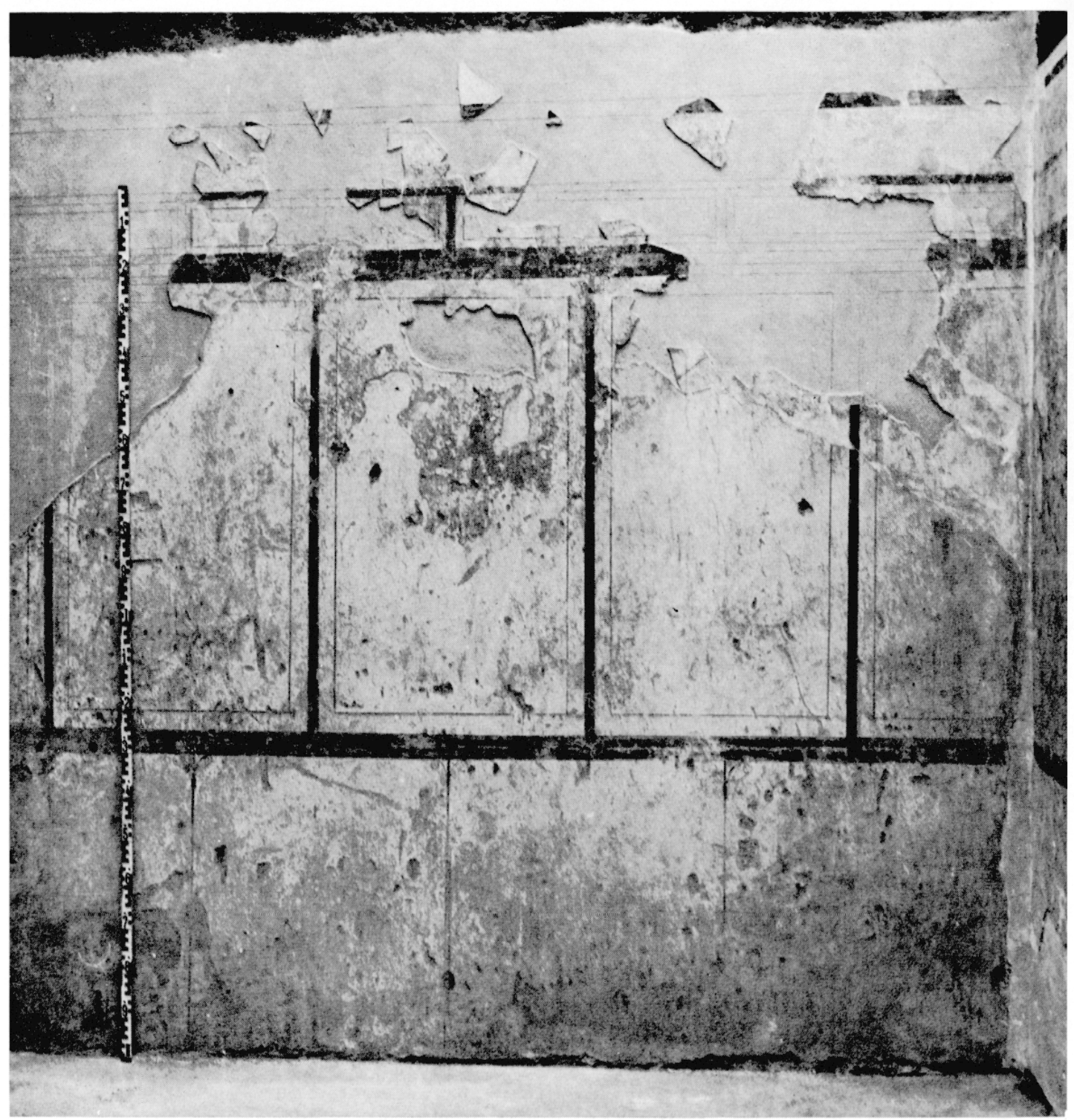

22 Rome, Palatin, maison des Masques et des guirlandes de pin, salle contiguë aux masques.

peint qui se recollent (inv. 1946. 204) ont été trouvés dans le silo $\mathrm{C}$ du chantier III. Ils présentent la moitié droite incomplète d'une volute à deux rubans noirs parallèles, avec bulbe central évidé; une bague à deux filets noirs également parallèles sépare les volutes de la hampe. Cette dernière semble avoir été composée de deux tiges noires parallèles, espacées de $1 \mathrm{~cm}$ environ. Le tout se détache sur le fond blanc de l'enduit (fig. 21).

Dans le même silo, d'autres trouvailles ont été faites, fragments de poterie grecque et campanienne, d'Arezzo, de Lezoux et de gobelets d'Aco. Beaucoup de silos à Ensérune. après avoir fait office de réservoirs, ont été convertis en dépotoirs dans la seconde moitié $\mathrm{du} \mathrm{I}^{\mathrm{er}}$ siècle av. J.-C. et les premières décennies de notre ère, car la construction de grandes citernes bâties apporta au problème de l'alimentation en eau une solution efficace et rendit 
les réservoirs inutiles ${ }^{19}$. Le fait de trouver ces fragments de peinture en même temps que des poteries d'époques diverses ne permet pas de leur assigner une date précise. La seule ('hose qui soit sûre est que la destruction, ou les changements intervenus dans la maison dont ils proviennent, n'ont pu se produire avant le milieu du I er siècle av. J.-C., date de la transformation des silos en dépotoirs. En effet, on imagine mal que des "gravats " soient précieusement conservés pour être jetés plus tard. Si les peintures peuvent être antéricures au milieu du ${ }^{\text {er }}$ siècle av. J.-C., les trouvailles de céramiques de Lezoux et de gobelets d'Aco nous indiquent que le dépotoir a servi jusqu'à une date avancée de l'époque romaine et que, par conséquent, les peintures pourraient en être contemporaines.

Nous pouvons done restreindre ce type de décor à la durée de l'occupation romaine à Ensérune, soit une période allant jusqu'au règne de Tibère. Les documents trouvés au Palatin, à Pompéi et à Masada vont nous confirmer la présence simultanée de cette sorte de peinture avec: le Second style pompéien et resserrer le laps de temps durant lequel elle a eu cours.

\section{Rome, le Palalin.}

a) Maison des "masques el des guirlandes de pins». -.- Au Palatin, sur une

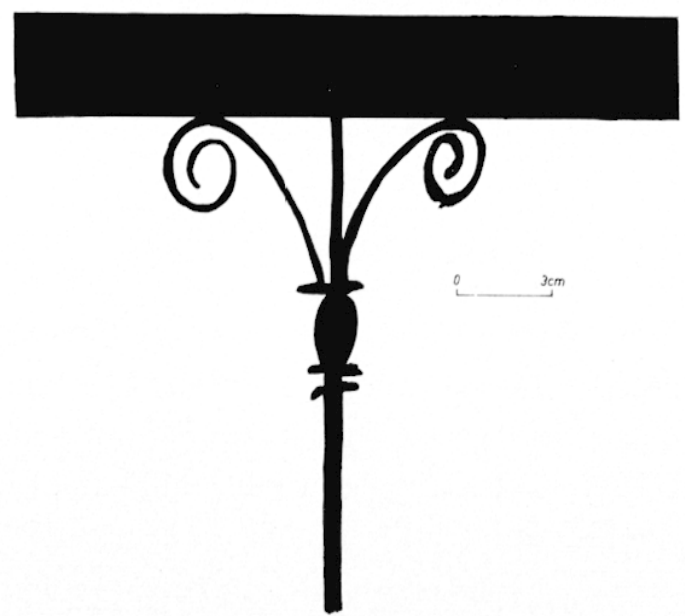

23 Rome, Palatin, maison des Masques et des guirlandes de pin, salle contigüe aux masques, détail d'une hampe à volutes. terrasse entaillée dans la pente de la colline, au-dessus du grand cirque, et à droite de la Scala Caci, M. G. F. Carettoni a fouillé une maison aux pièces peintes, dont deux de Second style qu'il a récemment publiées ${ }^{20}$. Une petite pièce secondaire, au nord-nord-est de la pièce peinte d'une architecture théâtrale ${ }^{21}$, conserve une peinture à fond blanc (hauteur totale $217 \mathrm{~cm}$ ), avec agencement de filets noirs et rouges et de volutes noires à hampes. L'ensemble est proche du décor de la maison aux deux alcôves (XVIII) à Glanum mais s'en distingue par quelques détails (fig. 22, 23).

La plinthe blanche, limitée à $70 \mathrm{~cm}$ du sol par une double bande noire et rouge, est rythmée par des tiges noires à volutes minces espacées de $67 \mathrm{~cm}$ en moyenne. Les volutes (de $9,5 \mathrm{~cm}$ à $13 \mathrm{~cm}$ de largeur) s'épanouissent au-dessus du bulbe de la tige, encadré de barbes, une au-dessus du bulbe, deux au-dessous.

(19) J. JavvoraY, Informations dans Gallia, XIV, 1956, p. 210.

(20) G. F. Caretroxi, Due nuovi ambienti dipinli sul Palatino, dans Bolleltino d'Arle, 1961, p. $189-199$.

(21) G. F. Cinettoxi, I problemi della zona auguslea del Palalino alla luce dei recenli scavi, dans Rendiconli della Ponlificia Accademia Romana di Archeologia, XXXIX, 1966-1967, fig. 3, plan, salles d. 
La bande rouge supérieure, horizontale, sert de bordure inférieure aux orthostates à fond blanc entourés d'une bande rouge (semblable sur les trois autres côtés) et agrémentés de traits d'encadrement intérieur (à $4,2 \mathrm{~cm}$ des bords). Sur l'un des orthostates, se trouve le dessin très efiacé d'un bateau, semble-t-il. Le couronnement se compose d'une bande noire horizontale, juxtaposée à la bordure rouge, d'un rang d'assises blanches $(12 \mathrm{~cm}$ de haut sur $80 \mathrm{~cm}$ de long) à bossages, dont les refends sont figurés par des bordures rouges et l'arête des bossages par des traits de même couleur. Au-dessus, un espace blanc est coupé par deux bandes noires (deux rangs d'assises, à nouveau?).

D'après des informations récentes ${ }^{22}$, la peinture est appliquée sur un mur en opus quadratum de tuf. Elle fait partie d'un groupe de locaux petits et modestement décorés d'un grand édifice établi sur la terrasse inférieure attenante au temple dit de Jupiter vainqueur et qui serait le temple d'Apollon construit à l'époque d'Auguste.

Deux pièces de ce groupe ont conservé de belles peintures qui se rattachent aux canons traditionnels du Second style architectonique et sont à dater de 50 à 25 av. J.-C. ${ }^{23}$. La première a des guirlandes de pins suspendues à de minces pilastres d'un péristyle avec ouverture à la partie supérieure sur d'autres péristyles, elle appartient à la phase IIa de Beyen ${ }^{24}$. La seconde, bâtie sur un schéma de décor de théâtre, avec au centre de chaque mur un sanctuaire champêtre, appartient à la phase II $\mathrm{b}^{25}$. Ainsi ce style de peinture à fond blanc est associé à des peintures de Second style allant de la première phase (structure fermée) à la phase de maturité, caractéristique du début du règne d'Auguste et qu'on retrouve dans la maison dite de Livie, sur le même Palatin. M. G. F. Carettoni avance l'hypothèse que ce serait cette demeure qui aurait appartenu à Auguste et qui fut construite peu après 36 av. J.-C. La structure des murs aussi bien que le type des mosaïques et des peintures conduit à dater l'ensemble de la première phase du règne d'Auguste.

b) Maison républicaine. - Dans le même secteur que "la maison des masques et des guirlandes de pins ", mais sous la terrasse supérieure attenante au temple de Jupiter vainqueur ou d'Apollon, nous avons retrouvé l'ordonnance supérieure de ces peintures à fond blanc, dans une maison que nous appellerons " républicaine " car elle remonte au dernier siècle de la République. On y rencontre aussi des peintures de la première phase du Second style. A gauche, avant d'entrer, le sommet des orthostates à fond blanc, limités par un trait rouge, avec traits d'encadrement intérieur noirs, est surmonté de deux rangs d'assises blanches à bossages dont les refends sont des traits noirs; l'arête des bossages est en traits rouges. La hauteur incomplète des orthostates est de $92 \mathrm{~cm}$ et leur largeur de $72 \mathrm{~cm}$, les assises ont $108 \mathrm{~cm}$ de long sur $26 \mathrm{~cm}$ de large. Les proportions, le traitement sont semblables à ceux de la pièce aux volutes de la maison des masques et des guirlandes de pins; l'existence d'une plinthe avec hampes à volutes, sans être attestée, peut être imaginée avec toute probabilité.

(22) Renseignements communiqués par M. G. F. Carettoni, que je remercie ici de son amabilité.

(23) G. F. Carettoni, op. cit., (n. 19), p. 68, n. 26.

(24) G. Bfyex, Die neuentdeckten Malereien auf dem Palatin, dans Bulletin von de Vereeniging tol bevordering der liennis van de anlieke beschaving te's Gravenhage, XXXIX, 1964, p. 140 et s.

(25) Analysé par G. PICARD, Revue des éludes latines, XLI, 1964, p. 382. 


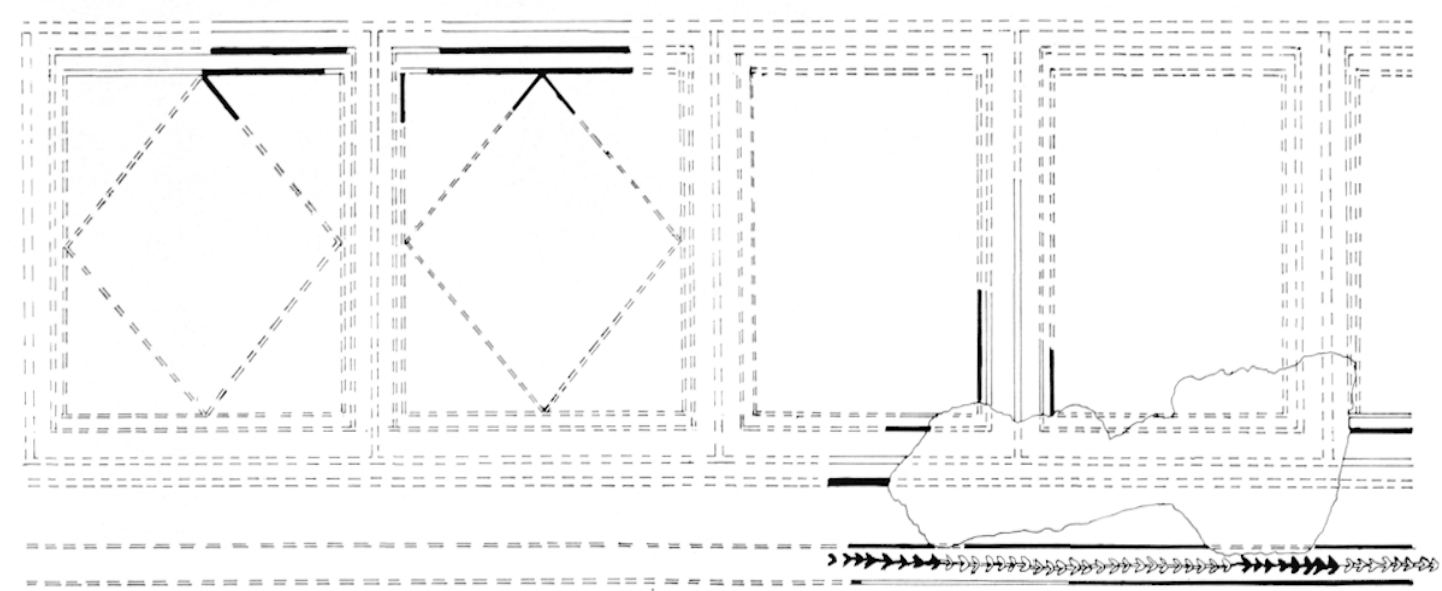

24 Pompéi (Reg. V, Ins. 1, 14).

Les pièces de cette maison sont décorées de peintures avec plinthe et orthostates imitant parfois le marbre, couronnés d'assises à bossages et d'entablement avec frise, moulures, denticules et modillons soutenant fictivement une corniche à caissons vus en perspective, tous éléments bien caractéristiques des débuts du Second style (entre 80 et 50 av. J.-C.). Ce voisinage entre des peintures à fond blanc, à hampes à volutes, et des peintures des débuts du Second style, nous le remarquons aussi à Pompéi.

Pompéi.

a) Région $V$, Insula 1, $n^{0} 14$. - Dans une petite pièce au fond, à gauche, sur un mur commun avec la rue voisine (vicolo di Mercurio), deux peintures superposées, très délabrées, ont attiré notre altention car la couche inférieure à fond blanc y rappelle le décor du mur I) de la maison aux deux alcôves (XVIII) de Glanum. La décoration à fond blanc a été piquetée pour permettre à l'enduit d'une seconde peinture, rouge, uniforme, d'être mieux accrochée.

Le fond blanc laisse encore deviner une plinthe limitée par un double filet noir horizontal à $68 \mathrm{~cm} \mathrm{du} \mathrm{sol} \mathrm{;} \mathrm{entre} \mathrm{les} \mathrm{deux} \mathrm{filets} \mathrm{parallèles,} \mathrm{se} \mathrm{développe} \mathrm{une} \mathrm{mince} \mathrm{tige} \mathrm{noire} \mathrm{feuillue} \mathrm{et} \mathrm{rigide.}$ Au-dessus, un filet noir et un filet rouge, espacés et parallèles, séparent la plinthe de cinq orthostates à fond blanc entourés de deux filets noirs (sur le petit còté supérieur) et de deux filets rouge et noir sur les autres côtés. Les deux orthostates de gauche reçoivent des losanges à trait noir (fig. 24).

La composition est malheureusement incomplète et aucun indice ne nous autorise à 
restituer des volutes noires sur la plinthe. Les comptes rendus de fouille n'apportent pas une grande lumière sur la question. On mentionne, dans la boutique du n ${ }^{0} 14$, une peinture du Second style tardif, très élégante ${ }^{26}$; un cubiculum à droite du tablinum, avec un décor dı dernier style, ainsi qu'un autre cubiculum à gauche de l'atrium, à fond blanc, de la dernière manière de Pompéi ; enfin, dans l'ala, une peinture du Quatrième style à fond noir. Aucune de ces peintures ne correspond, pour l'emplacement ou pour la description, à celle de cette pièce du fond, située à gauche. Faute d'un plan précis avec une numérotation des pièces, il est impossible de localiser ces décors, disparus maintenant. Nous pouvons seulement retenir que cette maison n'avait pas de peintures du Troisième style mais que le Second et le Quatrième y étaient exclusivement représentés. La superposition des deux peintures que nous avons remarquée nous fait supposer que le décor inférieur à fond blanc a toutes chances d'appartenir au Second style.

b) Maison des Noces d'argenl $(\mathrm{V}, 2, \mathrm{E})$. - La pièce f conserve une décoration à fond blanc, à la fois proche et différente de tous les exemples que nous avons vus jusqu'ici (fig. 25).

Le mur du fond (c'est-à-dire s.-o.), lorsque l'on pénètre dans cette pièce assez obscure et étroite, est le mieux conservé. Lne plinthe très basse ( $46 \mathrm{~cm}$ de hauteur), blanche, mouchetée de rouge, est, limitée par deux filets noirs parallèles très espacés. Entre ces deux filets, des rectangles très allongés (79 $\mathrm{cm}$ de long sur $13,5 \mathrm{~cm}$ de large $)$ sont disposés à intervalles légèrement irréguliers; le long de leurs petits còtés, à l'intérieur, sont appuyés des demi-cercles noirs (fig. 26).

Des hampes noires, distantes de $93 \mathrm{~cm}$ les unes des autres, jalonnent la plinthe; leurs têtes se terminent par un bulbe et une boule à piquants hérissés, qui montent jusqu'au milieu du double filet noir limitant la plinthe, entre les rectangles allongés (fig. 27). Partant des deux hampes centrales de la paroi du fond, deux guirlandes noires, attachées par des nouds, vont s'accrocher sur une hampe intermédiaire plus basse, plus mince. Celle-ci est ornée d'un nœud de ruban à mi-hauteur, son extrémité en forme de cloche renversée, noire, aboutit au filet inférieur de la limite de plinthe.

$\lambda$ u-dessus des filets séparatifs de la plinthe, de grands orthostates à fond blanc $(127 \mathrm{~cm}$ de haut, $102 \mathrm{~cm}$ de large) sont tracés au moyen de filets noir pour figurer les refends (dans le prolongement des hampes) et les traits d'encadrement intérieur. Au sommet, on croit distinguer une bande horizontale avec des rectangles d'assises de grand appareil (elle n'a pas été reproduite sur notre relevé).

Ce décor, fait uniquement de lignes noires sur le fond blanc de l'enduit, présente malgré cette économie de moyens une composition plus complexe que celles des autres peintures de la série. Les hampes sont des thyrses; bien qu'ils soient mal rendus, leur identification n'est pas douteuse ${ }^{27}$. Les nœuds de ruban, les guirlandes suggèrent, très loinlainement cerles mais sensiblement, des attributs dionysiaques. De plus, l'agencement des différentes parties du décor est curicux car il semble beaucoup plus proche du Premier style que du Second: la plinthe est très basse, conformément au style structural grec, à Délos par exemple, où. . Bulard note une hauteur moyenne de $30 \mathrm{~cm}$ contre 90 à $100 \mathrm{~cm}$ à Pompéi ${ }^{28}$. Autre indice plus intéressant et plus parlant, les longs rectangles allongés

(26) A. Mav, Bullellino dell' Instituto di Corrispondenza archeologica, 1877, p. 129)-134.

27) Voir notamment la mosaíque du cortège dionysiaque à ()lynthe, où la pomme de pin terminale du thyrse a la forme d'uni: boule de piquants, dans l'une des représentations.

(28) M. ButanI), Peintures murales el mosaïques de Délos, dans Monumenls... Piol, XIV, 1908, p. 170. 
au-dessus de la plinthe sont une transposition picturale des fameux bandeaux en relief qu'on rencontre à Délos, à cet emplacement même. Remarquons que ce bandeau n'est pas à section carrée comme sur la plupart des documents déliens ou pompéiens du Premier style, mais à section semi-circulaire comme s'il s'agissait d'un demi-tore. Nous avons son correspondant en relief sur une peinture du Premier style à Priène ${ }^{29}$.

Ce spécimen de décor à fond blanc, qui apparaît comme intermédiaire entre le Premier et le Second style, est daté avec une assez bonne précision. La maison des Noces d'argent est une demeure grandiose dont la construction remonte à l'époque samnite; presque tout son décor est du Premier et surtout du Second style ${ }^{30}$. Dans l'état actuel du plan, quatre petites pièces, deux sur chaque côté (sans tenir compte des alae), s'ouvrent sur l'alrium. (fig. 25). Cet atrium tétrastyle de l'époque préromaine avait cette particularité qu'à l'origine un étage supéricur n'avait pas été prévu. Les pièces avaient une hauteur énorme et dans ce premier aménagement les escaliers $g$ et $k$ manquaient. $g$ faisait

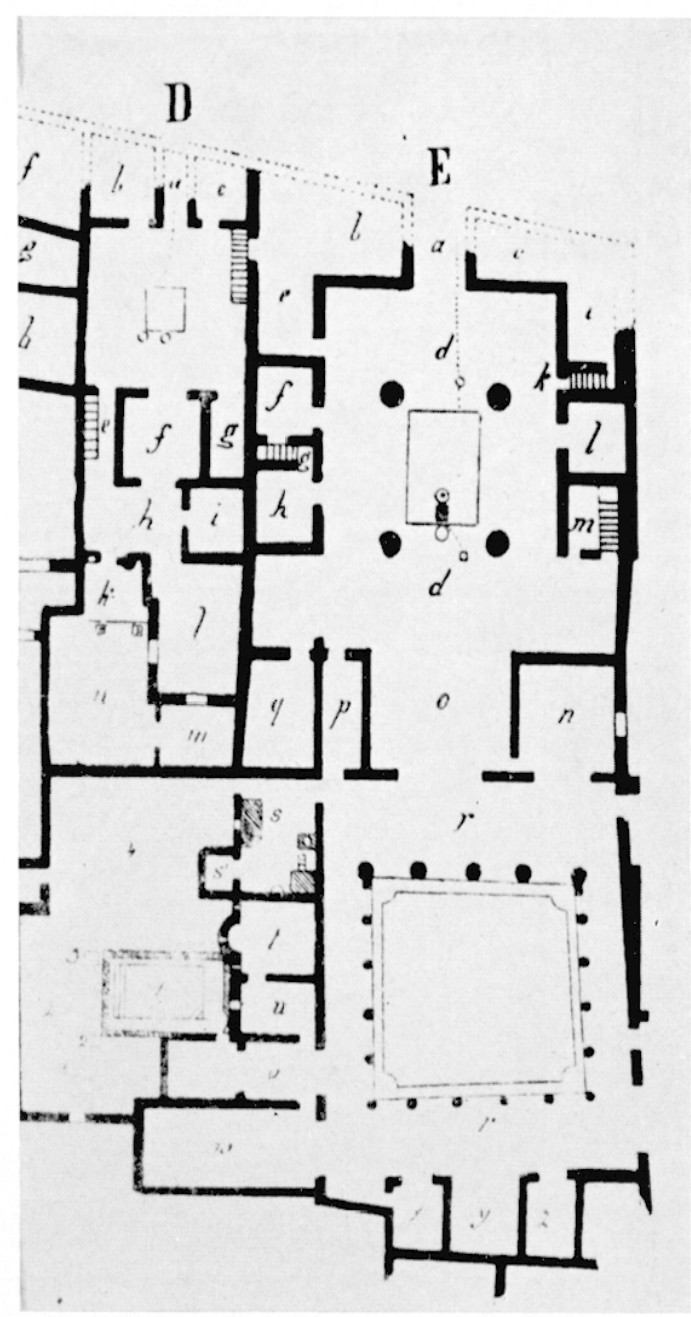

25 Pompéi, plan de la maison des Noces d'argent IReg. V, Ins. II, E). alors partie de $h$; on y trouve quelques rares traces du Premier style, ainsi qu'en $f$, selon A. Mau ${ }^{31}$. Dans les derniers temps du Second style décoratif, c'est-à-dire dans les premières années du règne d'Auguste, la maison et en l'espèce l'alrium et ses pièces adjacentes furent transformées. Les chambres latérales furent réduites à des petits locaux bas avec étages supérieurs, en prenant entre deux pièces la place des deux escaliers $k$ et $g$. Les transformations achevées, la maison fut décorée en peintures de Second style (dernière phase), par exemple sur les murs de l'alrium, des alae, des salles $v, x, y, z$. On montait aux pièces supérieures du côté droit de

(29) Cf. M. Borda, La pittura romana, Milan, 1958, photo p. 14.

(30) A droite du tablinum, il y avait une seule salle à l'origine, comme de l'autre cỏté ; le mur de séparation entre le couloir $p$ et la pièce $q$ est postérieur et le décor de cette dernière appartient au Troisième style à candélabres, cf. A. Mav, Scavi di Pompei, ins. V. 2, dans Mitteilungen des Kaiserlich deutschen Archaenlogischen Instiluls, Römische abteilung, 8, 1893, p. 29-30.

(3I) A. MAU, op. cil., p. 28. 


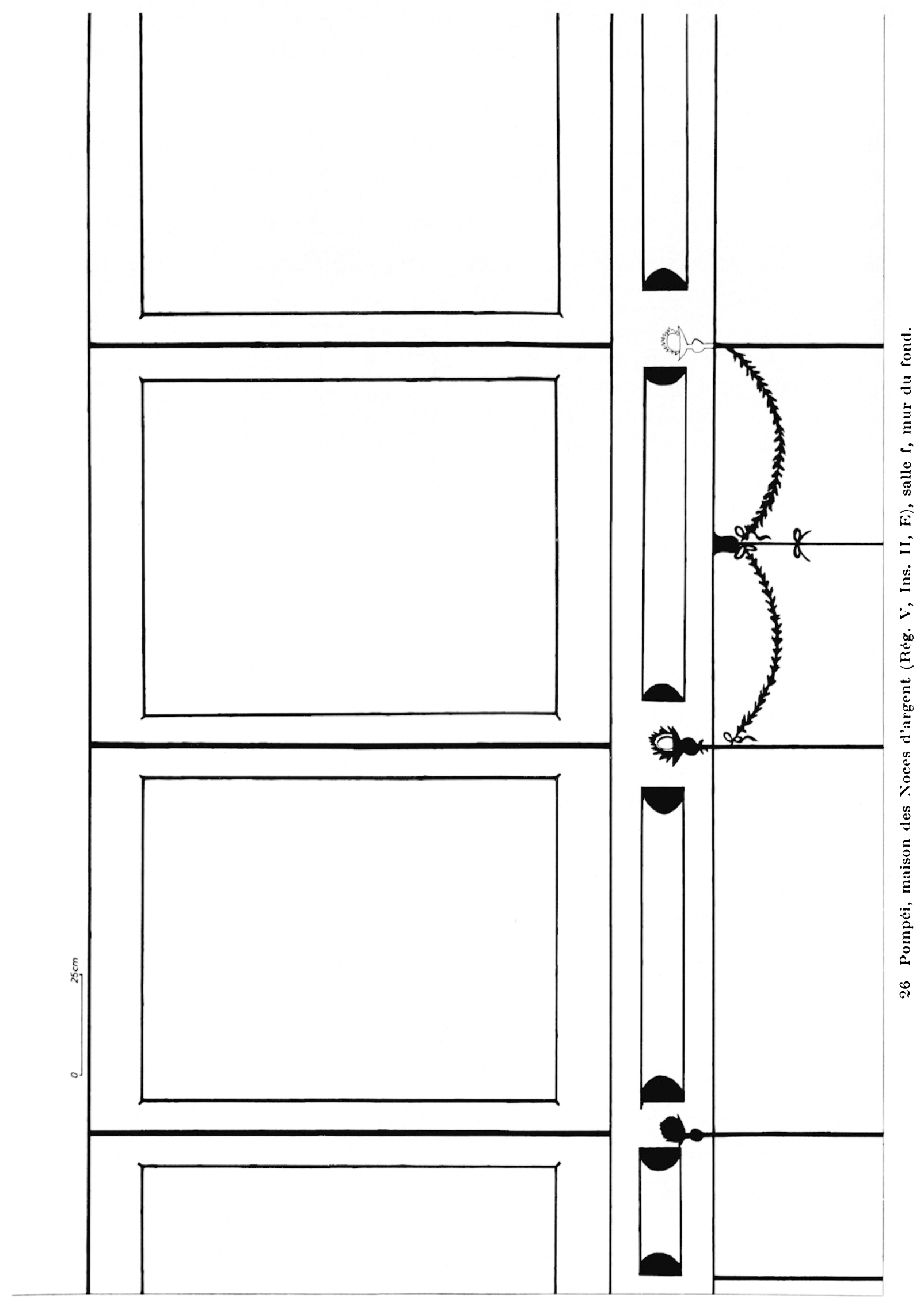


l'atrium (s.-o) par l'escalier $g$, accessible par la pièce $f$, celle qui renferme justement ce décor à fond blanc avec thyrses. Cet escalier a un décor de Premier style à stucs en relief ; la plinthe est rouge bordeaux (112 cm de haut), le bandeau en relief qui la limite est horizontal et ne suit pas la ligne ascendantc de l'escalier. Ce détail nous montre qu'effectivement le décor de Premier style a précédé la construction de l'escalier et qu'il ne lui est pas adapté.

Quant à la décoration de la pièce $f, \mathrm{~A}$. Mau reprend plus loin sa remarque de la page 29 où il parlait des rares vestiges du Premier style : «f, ou plutôt $g$, [c'est-à-dire uniquement la cage de l'escalier] et $h$ ont des restes du Premier style ${ }^{32}$ ». L'auteur de la chronologie des styles pompéiens passe donc sciemment sous silence ce décor de la salle $f$, car il ne cadrait pas facilement avec son classement; il le distingue bien du Premier style, puisqu'il rectifie ce qu'il a dit précédemment, mais il ne le range pas non plus dans le Second. Cette transformation du volume intérieur des pièces latérales de l'atrium et la décoration de Second style qui s'en suivit, datant du débul du règne d'Auguste, nous donnent du même coup la date du décor de la salle $f$, qui est contemporaine de cette période. Nous sommes donc sûre que ce type de décor à fond blanc est postérieur au

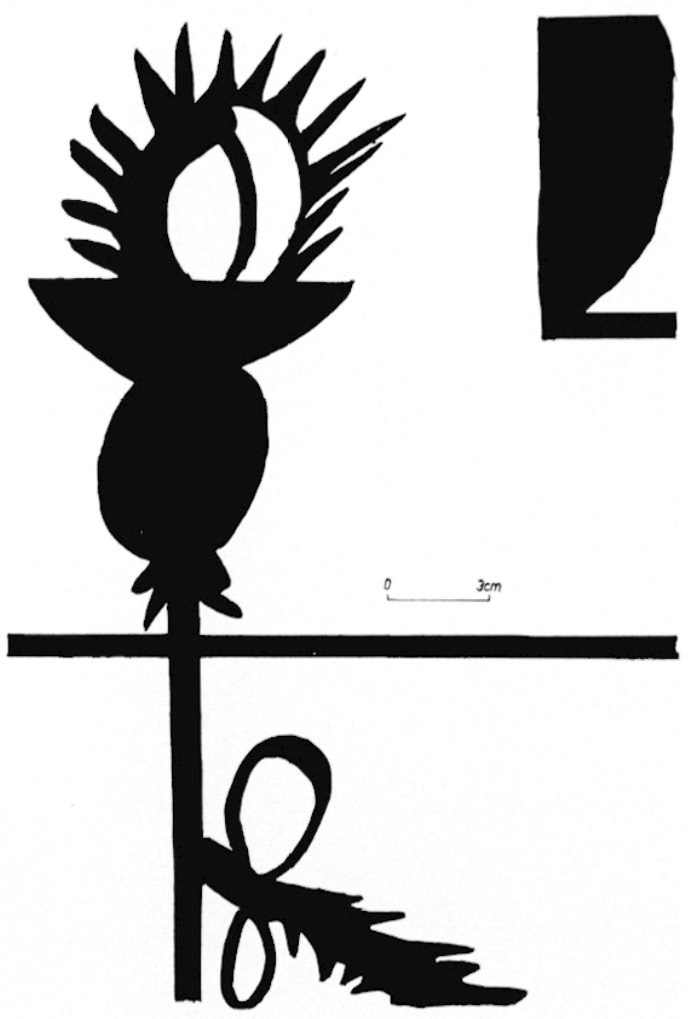

27 Pompéi, maison des Noces d'argent (Rég. V, Ins. II, E), salle f, détail.

Premier style présent dans l'escalier $g$.

Après étude de ces derniers documents nous présumons qu'il s'agit d'un style schématique, réalisé avec une grande économie de moyens, réservé à des pièces de peu d'importance, presque des réduits, se développant parallèlement au Second style "classique " c'est-à-dire polychrome et architectonique. Il semble bien être contemporain de la dernière phase du Second style, comme nous le suggèrent la maison des Masques et des guirlandes de pins sur le Palatin et la maison des Noces d'argent $(\mathrm{V}, 2, \mathrm{E})$ à Pompéi. Si nous adoptons la datation habituelle des styles pompéiens, nous pouvons fixer la présence de ce "Second style schématique » au début du règne d'Auguste ; c'est la date que les peintures de Masada nous donnent également.

\section{Masada (Palestine).}

Palais d'Hérode. - Masada est une forteresse située près de la rive ouest de la Mer morte, au sud. D'abord occupée par les rois juifs, elle fut transformée en véritable citadelle

(32) A. MAN o. c. p. 38. 


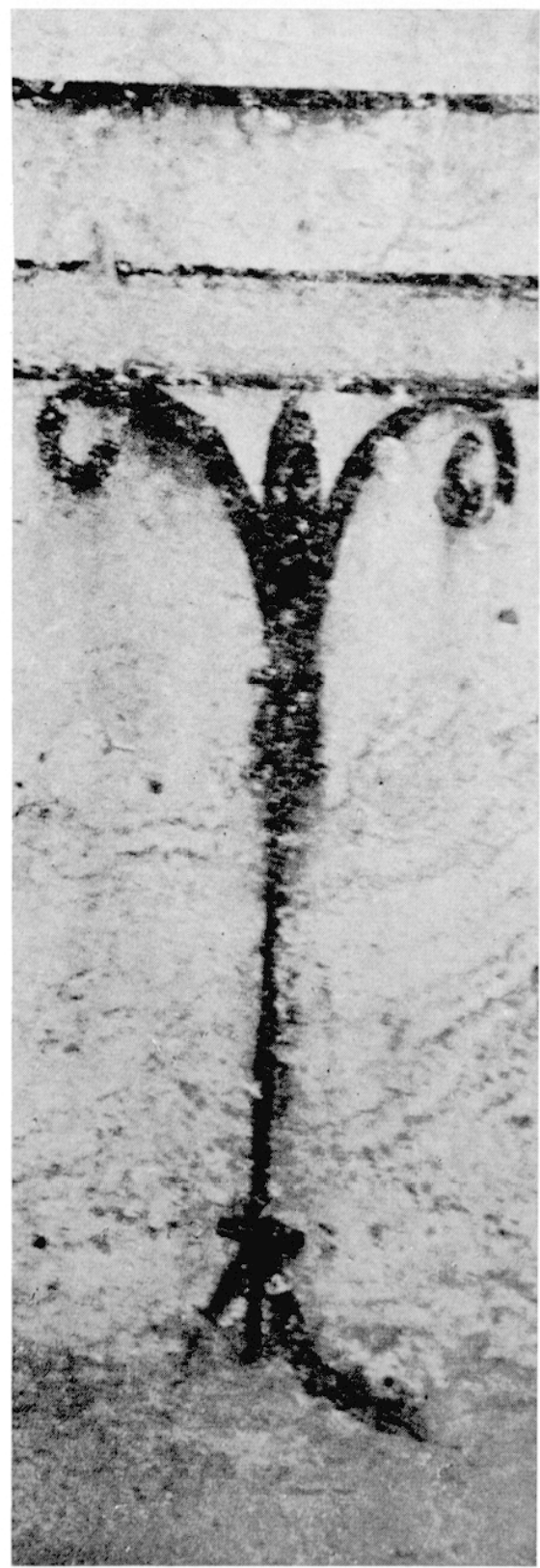

28 Masada, palais du nord (palais d'Hérode) pièce 15 , hampe à volutes. royale par Hérode ${ }^{33}$. Après la mort de celui-ci, une garnison romaine s'y installa, qui fut expulsée en 66 ap. J.-C., mais Masada retomba sous la domination romaine en 73 .

C'est le palais du nord qui contient les peintures les plus intéressantes, il est construit en trois terrasses ; la terrasse inférieure, rectangulaire, présente un espace central environné de deux rangs de colonnes avec des peintures sur les parlies basses. Dans la pièce 1, le coin ouest portait des peintures dont tous les panneaux tendaient à imiter la pierre et les veinures du marbre ${ }^{34}$. Dans la pièce 5 , le mur sud du portique méridional était peint, lui aussi, de panneaux entre les demi-colonnes engagées; toujours la même composition d'un compartiment étroit et d'un compartiment allongé, flanqués de compartiments plus larges de chaque còté ${ }^{35}$. Le peintre a essayé de créer une illusion de profondeur en utilisant une fausse façade. Les compartiments de part et d'autre d'une colonne sont de la mème couleur et donnent l'impression qu'il y avait un mur derriere elle. Le compartiment central entre deux colonnes imite les veinures d'un marbre.

Ciette description correspond à des peintures de Second style à structure fermée, très simple; or elles sont liées à une peinture différente, subsistant dans la pièce 13, dans le coin s.-o. La partie supérieure est divisée en quadrilatères par des encadrements noirs tandis que la partie inférieure est décorée par un rang de "palmiers nains" ou de "palmettes" stylisés ${ }^{36}$ (fig. 28). Ces palmiers ou palmettes sont en fait des hampes noires terminées par des volutes se détachant sur un fond blanc. Un détail important distingue ce document de toute la série : la basse de la hampe est garnie d'un trait horizontal et de deux pédoncules se recourbant latéralement, comme s'il s'agissait d'un trépied. La hampe est très courte ( $30 \mathrm{~cm}$ environ).

Le palais du nord a été construit pendant le règne d'Hérode, qui commence réellement en 37 av. J.-C. ; Y. Yadin pense qu'il a été bâti entre 36 et 25 avant J.-C. et qu'il a été définitivement détruit en 73 ap. J.-C. Le palais a dû être décoré entre 36 et 25 av. J.-C.,

(33) Yigael Yanix, Masada, Herod's Forlress and the Zeaiol's lasl Stand, Londres,1966, p. 16.

(34) Avi-Yovali, N. Avigad, Y. Aharowi, I. Dunayfwsky, S. Gutmax, The survey and excavalion of Masada, 1955-1956, dans Israël exploralion Journal, 1957, p. 1-60.

(35) Y. Yadis, The excavation of Masada, 1963-1964, ibid., 15, 1965, p. 11 a 14

(36) "Palmetto" en anglais peut vouloir dire palmiers nains ou palmettes. 
laps de temps qui concorde bien avec le type des peintures rencontré et avec les dates fournies par les autres sites qui connaissent ce style schématique.

A cet ensemble de peintures formant un tout assez cohérent viennent s'agréger de façon plus malaisée certains fragments d'enduit peint gallo-romains de Martizay.

\section{Martizay (Indre).}

Au lieu-dit St-Romain, à la sortie ouest du bourg de Martizay, sur la rive droite de la Claise, ont été trouvé des fragments de peinture murale à fond blanc présentant, sous une large bande noire, une hampe brune, barrée de trois traits, à volutes de même couleur, issues d'un bulbe terminant la hampe (fig. 29). Apparemment, la ressemblance est nette avec les autres documents précédemment analysés. En fait, un examen attentif des fragments rèvèle une technique assez différente; de plus, le contexte archéologique parait peu conciliable avec une date aussi précoce que le règne d'Auguste.

Dans la pièce $\mathrm{P} 3$, où l'on a recueilli ces hampes à volutes, sur le mur M 5 , une portion d'enduit mural est conservé ; il représente une hampe jaune, verticale. La plinthe avait donc un dispositif de hampes, peut-être couronnées de volutes. La poterie du site attesterait une occupation dès le milieu du I $^{\text {er }}$ siècle av. J.-C.,

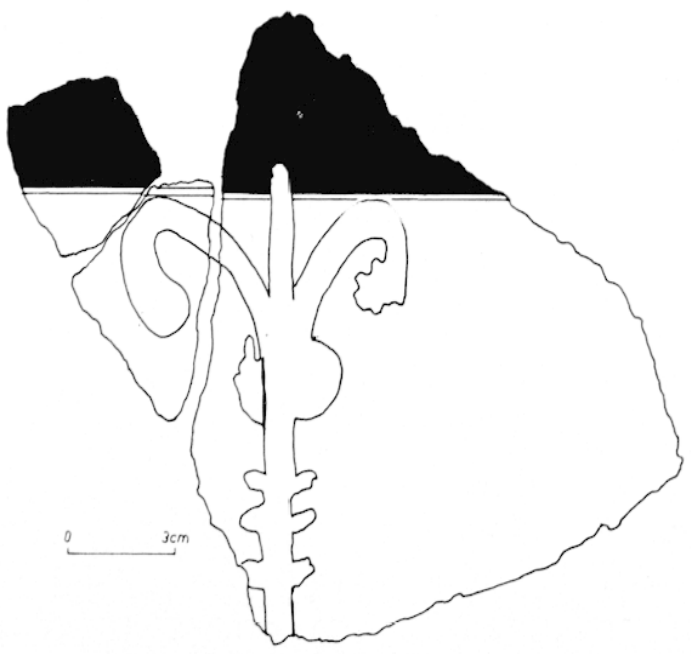

29 Martizay (Indre), fragments d'enduit peint provenant de la pièce P 3 (Inv. nº 65 P 3. 35). notamment de la poterie jaune clair, avec un décor à la roulette romain primitif (50 av. J.-C.), d'après le rapport de M. J.-L. Soubrier ${ }^{37}$; le reste de la poterie trouvée est plus tardive.

La restauration encore en cours des peintures de Martizay nous a montré qu'il y avait deux couches superposées d'enduits peints, la couche supérieure concernant les hampes à volutes. La couche inférieure, soit directement posée sur une terre tassée qui s'effrite énormément, soit étendue sur une couche de mortier assez grossier, est à fond blanc orné, semble-t-il, de motifs végétaux jaunes et rouge violacé ; les témoins conservés sont trop peu nombreux et trop exigus pour qu'on puisse se faire une idée de leur style. Rien de géométrique, en tout cas, pouvant faire songer au Second style de Pompéi. Sur certains morceaux le piquetage, pour assurer une meilleure prise à la nouvelle peinture, est visible.

La nouvelle peinture, celle des hampes à volutes, est donc postérieure de quelques années ou de plusieurs à la première, dont on ne peut fixer la date mais dont on doit souligner le caractère fruste. Cette peinture supérieure est associée à des motifs végétaux divers,

(37) J.-L. Soubrier, rapport sur les fouilles de 1961, p. 12 (exemplaire dactylographié). 
peints en ocre jaune et en ocre rouge. On notera une lourde guirlande de fruits, assez sommairement exécutée, des tiges végétales dont le lien ne peut plus être établi par une reconstitution d'ensemble car tous les fragments blancs sans décor, découverts au cours de la fouille, n'ont pas été gardés.

Pour établir la date de ce décor de hampes à volutes, les éléments fournis par la stratigraphie et par les fouilles ne sont pas décisifs. On avait pensé que cet édifice était tardif, du ${ }_{I I}{ }^{e}$ et du ${ }_{I I I}{ }^{e}$ siècle ap. J.-C. ${ }^{33}$. La découverte de deux pavages successifs dans la pièce $\mathrm{P} 3$-- les peintures à deux couches superposées appartenant à l'état premier de l'habitat, sur le premier pavage - montre une occupation très longue. Il y a même des peintures contemporaines du second pavement, mais trop peu nombreuses pour qu'on puisse savoir à quelle période les rattacher.

Restent les arguments stylistiques. Certes, le motif est proche de la série déjà étudiée mais la technique en diffère. Les couleurs n'ont pas été étendues sur le fond blanc de l'enduit même, comme pour les exemples précédents, mais sur une couche épaisse de couleur blanche, appliquée au préalable sur l'enduit, très grossièrement car les raies tracées par les poils du pinceau sont visibles. Afin d'avoir des points de repère, au moment de fixer la composition d'ensemble et l'emplacement des hampes et de la bande noire qui la surmonte, une ficelle enduite d'une poudre d'ocre rouge a été tendue sur l'enduit peint de frais; elle a laissé de profonds sillons colorés de rouge. Ce procédé est bien connu ${ }^{39}$. Le décor est associé à une guirlande ; G. Picard a songé à la comparer aux guirlandes de Second style augustéen. Quant aux autres décors végétaux, par leur caractère frustre, ils se rapprochent des fragments trouvés à Ensérune, dans la maison aux pseudo-thyrses (II, 7) (fig. 19). Étant donné la subjectivité de critères uniquement stylistiques, fondés sur des documents très fragmentaires, il serait imprudent de conclure fermement à un décor d'époque augustéenne, encore que rien ne s'y oppose. Il faut attendre que la fouille soit terminée, que toutes les reconstitutions possibles soient achevées. Notons simplement la parenté iconographique, sinon stylistique, absolument indiscutable.

Cette revue de tous les sites, avec leur chronologie, établie d'après des données de fouilles et d'histoire, a été reportée sur le tableau ci-après. On est immédiatement frappé par cet intervalle de temps compris entre 40 et 25 av. J.-C., valable pour tous les sites. Ces dates sont vérifiées par l'association de ce style schématique avec des peintures des deux premiers styles. Ainsi, on peut supposer avec vraisemblance qu'au début du règne d'Auguste, alors que le Second style allait vers son raffinement final, un Second slyle schémalique existait qui annonce déjà certains caractères du Troisième style. Un goût pour un fond clair, neutre, blanc, une décoration ténue, collée à la paroi qui n'a plus que des vélléités de profondeur, tout cela, une dizaine d'années plus tard, s'impose peu à peu. Ce n'est pas l'un des moindres chefs d'intérêt de ce type de décor, qui reste pourtant bien humble.

(38) F. EyGun, Gallia, XX1, 1963, p. 433-484.

(39) Par exemple à Zliten, cf. S. Aurigemma, Tripolitania, i monumenti d'arle decorativa, II, Le pilture d'età romana, Rome, 1962, p. 42. 
PEINTURES DE SEGOND STYLE "SGHÉmatiQUE"

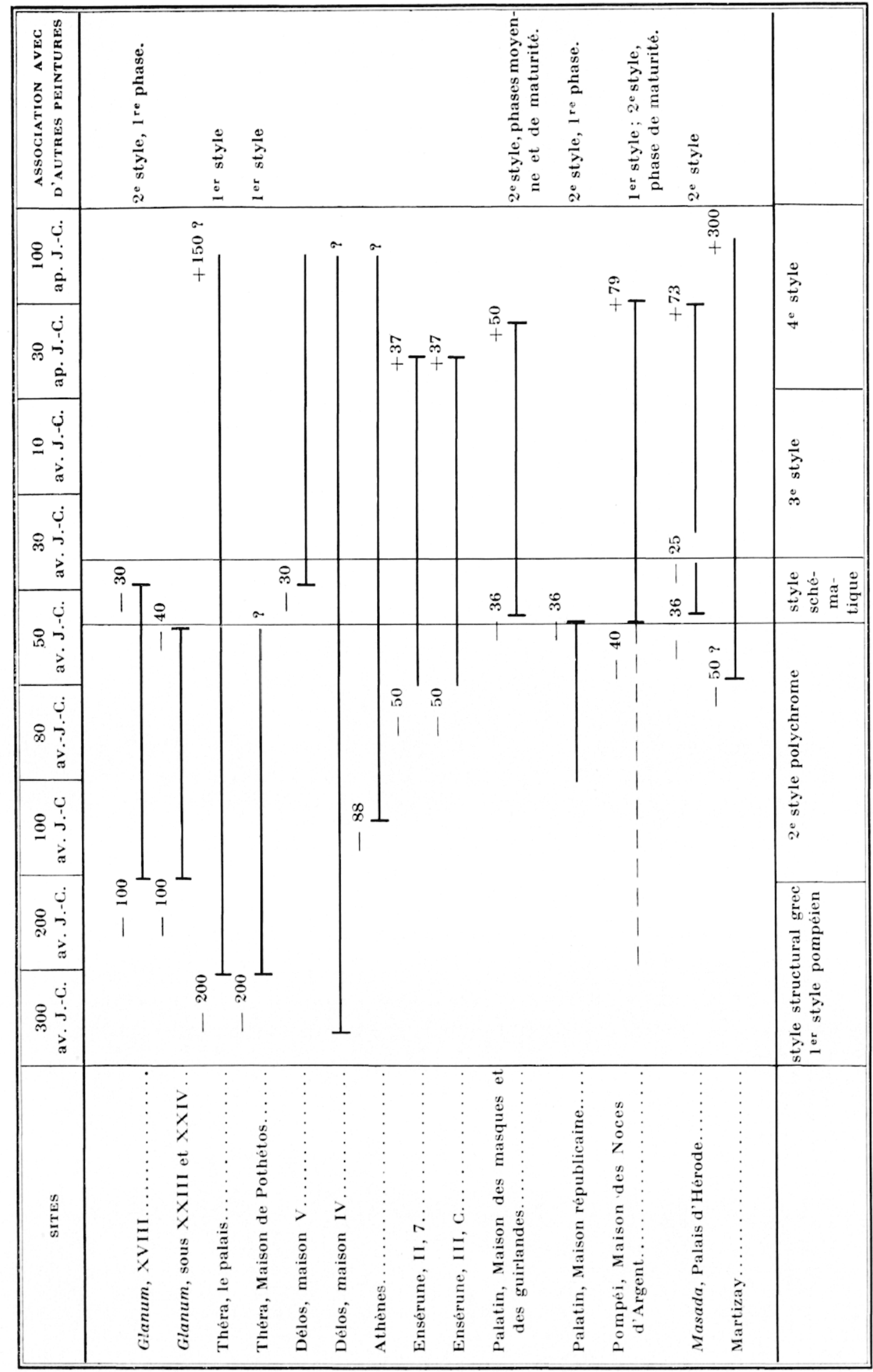


Significalion des hampes à volules.

Selon les exemplaires de volutes, le caractère floral est plus ou moins accentué. Il faut mettre à part la peinture de la pièce $f$ de la maison des Noces d'argent $(\mathrm{V}, 2, \mathrm{E})$ à Pompéi, qui représente des thyrses. De même à Délos, dans la chambre $m$ de la maison $V$ et à Théra, dans la salle $\mathrm{G}$ de la maison de Pothétos, certaines hampes font songer à des tridents végétalisés plutôt qu'à des motifs purement floraux. Dans les salles $\mathrm{S}$ et $\Theta$ du palais de Théra, certaines hampes rappellent le caducée de Mercure, avec double croisement des volutes vers l'intéricur.

Peut-on penser qu'à l'origine les artistes voulaient représenter les attributs de trois grands dieux grecs : le trident de Neptune, le thyrse de Dionysos et le caducée de Mercure ? Quoi qu'il en soit, c'est en territoire grec et surtout parmi les exemples qui paraissent les plus anciens, en connexion avec des restes de peintures du Premier style, que l'on trouve ces pseudo-attributs religieux.

Ailleurs, au Palatin, à Glanum, à Ensérune, à Masada et à Martizay, où il y a association avec des peintures du Second style, il s'agit uniquement de volutes. L'apparence florale de ce groupe qui paraît postérieur au précédent ne serait-elle pas le résultat d'une "végétalisation " de motifs, non floraux à l'origine (peut-être des attributs divins), dont le sens se serait perdu?

Pour les peintures de la maison aux deux alcôves (XVIII) de Glanum, M. H. Rolland $\mathrm{y}$ voit une vague stylisation de volutes ioniques ${ }^{40}$. Ce rapprochement nous parait peu fondé parce que l'emplacement de ces colonnettes à chapiteaux ioniques, schématisées à l'extrême, serait insolite. En effet, le sommet du décor mural se termine par un entablement avec frise, modillons et moulures très simplifiées. S'il s'agissait de colonnettes, même linéaires, elles auraient pris place entre les orthostates, les volutes venant s'appliquer sous la frise de l'entablement; or, ce n'est pas le cas, pas plus qu'au Palatin, dans la maison des Masques et des guirlandes de pins.

Il vaut mieux s'orienter du côté d'une origine florale du motif, dont le modèle semble difficile à retrouver, si jamais une fleur bien précise a servi de point de départ ${ }^{41}$. En ce qui concerne le décor, plus ténu, situé sous la cour des temples géminés (XXIII et XXIV), il ne s'agit plus d'imitation de fleur.

En fait, ces volutes n'ont aucune prétention à la copie du réel. Visiblement schématisées, elles sont le fruit d'une longue tradition artistique. La schématisation très poussée, de la plante permettrait toutes sortes d'hypothèses ct notamment celle d'une parenté avec le lotus égyptien. Cette suggestion n'est pas dénuée de fondement lorsque l'on observe la mode du paysage à l'égyptienne dans la peinture du second style mais en l'absence de points de comparaison certains, on ne peut travailler sur une telle hypothèse.

40) H. RollaNd, Gallia, XVI, 1958, p. 108.

(41) D'après M. M. Lecompte, botaniste au Muséum d'histoire naturelle, l'allure générale des volutes évoque le lis blanc (lilium candidum) mais la représentation proposée par le peintre n'est pas exacte. Le toupet situé au-dessus du bulbe pourrait être les stigmates, le bulbe lui-même, l'ovaire et les volutes, la corolle ; mais les stigmates et le bulbe ne se présentent pas de cette façon sur un lilium: il s'agit incontestablement d'une fleur mais composite ou imaginée à partir d'une fleur réelle. Le lis est la fleur qui, néanmoins, se rapproche le plus du motif. 
Cependant, au Palatin, à Glanum, à Ensérune, ces volutes sont nettement décoratives, elles n'ont presque plus rien à voir avec le monde végétal (à Athènes et à Délos, l'inspiration vient plutôt d'une tige ornée de feuilles que de la corolle d'une fleur). Leur présence sur la plinthe est insolite car celle-ci est le support d'un ordre architectonique de pur Second style, avec orthotastes, assises d'appareil, modillons et entablement. Quel est leur rôle dans cette composition si bien ordonnée ? Le document de Masada nous donne, semble-t-il, la clef du problème. Jés hampes à volutes de Masada sont des sortes de trépieds, de candélabres. Les traits horizontaux sous les volutes et à la base du pied serviraient à figurer les bagues nécessaires pour ajuster les différentes pièces métalliques. L'emplacement, les proportions sont d'un candélabre ${ }^{42}$.

Pour Glanum et Ensérune, on songe plus spécialement à une barrière, une clôture. En eflet, dès le début du Second style, on trouve peints sur des plinthes des treillis de jardin en losanges ou des écailles de claustra, comme on en voit dans la maison des Griffons sur le Palatin ${ }^{43}$. Plus tard, on figure sur des plinthes de petites balustrardes en croix de St-André, à Trèves ${ }^{44}$, à Glanum même, dans la maison des Antes (VI) ${ }^{45}$ et à Fauroux (Tarnet Garonne $)^{46}$. Il s'agit bien de barrières car des clôtures de mème type avaient servi auparavant, dans la peinture pompéienne, à fermer la partie basse des arcades peintes dans les architectures supérieures.

Il ne faut pas confondre ce style à fond blanc, parallèle au Second style, avec d'autres styles postérieurs, également à fond blanc. Par exemple nous voyons fréquemment à Pompéi un parallèle schématique du Troisième style. Le fond est entièrement blanc, les filets d'encadrement sont rouges et noirs, parfois jaunes et très souvent, au centre des panneaux, un petit tableau quadrangulaire porte une nature morte ou un oiseau becquetant un fruit. Il est impossible de confondre avec le Second style schématique que nous avons analysé. Il n'y a jamais de hampes à volutes, pas d'orthostates ornés de losanges, pas d'assises d'appareil. Au contraire, il s'agit d'un style-tapis très simple. Nous en avons des exemples même dans la maison des masques et des guirlandes de pins sur le Palatin, dans une pièce qui a été refaite au début du ${ }^{\text {er }}$ siècle ap. J.-C. et qui se trouve en face de la salle aux guirlandes de pins. La plinthe blanche est limitée par un trait noir ainsi que les panneaux qui tiennent lieu d'orthostates; une guirlande traverse le panneau de gauche, un masque y est suspendu. A droite, c'est un perroquet qui anime le centre. Sur le mur d'en face, un oiseau becquette des baies. Nous sommes là en présence d'un Troisième style linéaire.

Cette notion de style schématique nous paraît importante car elle ressurgit à différentes époques; elle est souvent parallèle à un style plus riche, plus coloré. W. Drack ${ }^{47}$ considère que les peintures à fond blanc, qui constituent la majeure partie des peintures murales romaines de la Suisse et qui sont tardives, ont leur point de départ dans le style "phil-

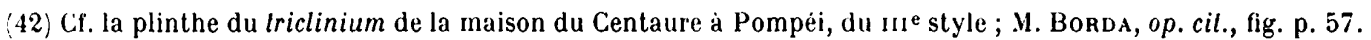

(43) G. E. Rızzo, Pillure della casa dei Grift, dans Monumenli della piltura antica scoperti in Italia, sect. III, fasc. 1 , Rome, 1936 , pl. C, VI.

(44) Steiner, Römische Wandmalerei in Trier, dans Trierer Zeilschrift, 1927, p. 54-68, fig.

45) H. Rollaxd, Fouilles de Glanum, Ier supplément à Gallia, fig. 66 .

(46) M. Labrolsse, Gallia, IX, 1951, p. 126-140, fig. 11.

(47) W. Drack, Die Romische Wandmalerei der Schweiz, Bâle, 1950, p. 27-29. 
hellène " de l'époque d'Hadrien. Ce genre s'est poursuivi avec des fortunes diverses jusqu'au Ive siècle. Pour le savant suisse, il serait peut-être caractéristique de la province par rapport à l'Italie. C'est ainsi qu'au III siècle, probablement, nous trouvons ces peintures à fond blanc, avec plinthes occupées par des barrières en croix de St-André auxquelles nous avons déjà fait allusion pour des documents de Glanum (maison des Antes), de Trèves, de Fauroux ; nous devons y ajouter Martigny, en Suisse. J. Jannoray avait confondu ce style très tardif avec le Second style schématique en rapprochant les volutes d'Ensérune des barrières en croix de St-André de la maison des Antes (VI) de Glanum ${ }^{48}$.

Il est certain que nous pourrions tirer des conclusions plus étoffées si les peintures murales des provinces de l'Empire romain et d'Italie avaient fait l'objet d'inventaires complets. Nos points de référence sont forcément restreints par la difficulté de rassembler la documentation, surtout lorsqu'il s'agit de peintures sans personnages et le plus souvent fragmentaires, jamais publiées. Pourtant les enseignements qu'on peut tirer de telles études sont encourageants et il faut espérer que ce domaine de l'archéologie prendra peu à peu la place qui lui revient. Nous serions très reconnaissante à ceux qui voudraient bien nous signaler éventuellement d'autres exemples de peintures de ce type ${ }^{49}$.

Alix BARBET.

(48) J. Jannohay, op. cil., p. 14, n. 1, référence à la salle L et 11 de la maison des Antes à Glanum.

(49) Les photographies et les relevés sont de l'auteur sauf : fig. 3, aimablement communiquée par $\mathrm{H}$. Rolland; fig. 10, d'après Théra, t. III, pl. 2 ; fig. 11, d'après Théra, III, pl. 1; fig. 12, d'après Théra, III, pl. 4; fig. 13, d'après Délos, Il, fig. 98 ; fig. 14, aimablement communiquee par Ph. Bruneau, de l'École française d'Athènes; fig. 15, d'après Théra, III, pl. 4 et d'après Délos, II, fig. 101 ; fig. 16, d'après J. JannoraY, Ensérune, fig. 10 ; fig. 22, aimablement communiquée par G. F. Carettoni ; fig. 25, d'après A. MAv, loc. cit., pl. 1 ; fig. 28, aimablement communiquée par Y. Yadin.

Nous remercions les éminents archéologues qui ont bien voulu nous prêter ces documents et dont nous venons de citer les noms, ainsi que M.M. G. Picard et H. Stern, qui nous ont conseillée dans notre recherche, M. Gallet de Santerre, M. le chanoine Giry, M. J.-L. Soubrier qui nous ont facilité l'accès des documents à Ensérune et à Martizay. 